\title{
OPEN A class III WRKY transcription factor in sugarcane was involved in biotic and abiotic stress responses
}

\author{
Dongjiao Wang ${ }^{1}$, Ling Wang ${ }^{1}$, Weihua Su ${ }^{1}$, Yongjuan Ren ${ }^{1}$, Chuihuai You ${ }^{1,2}$, Chang Zhang ${ }^{1}$, \\ Youxiong Que ${ }^{1,3 \bowtie}$ \& Yachun $\mathrm{Su}^{1,3 \bowtie}$
}

WRKY transcription factors play significant roles in plant stress responses. In this study, a class III WRKY gene ScWRKY5, was successfully isolated from sugarcane variety ROC22. The ScWRKY5 was a nucleus protein with transcriptional activation activity. The ScWRKY5 gene was constitutively expressed in all the sugarcane tissues, with the highest expression level in the stem epidermis and the lowest in the root. After inoculation with Sporisorium scitamineum for $1 \mathrm{~d}$, the expression level of ScWRKY5 was significantly increased in two smut-resistant varieties (YZ01-1413 and LC05-136), while it was decreased in three smut-susceptible varieties (ROC22, YZ03-103, and FN40). Besides, the expression level of ScWRKY5 was increased by the plant hormones salicylic acid (SA) and abscisic acid (ABA), as well as the abiotic factors polyethylene glycol (PEG) and sodium chloride ( $\mathrm{NaCl}$ ). Transient overexpression of the SCWRKY5 gene enhanced the resistance of Nicotiana benthamiana to the tobacco bacterial pathogen Ralstonia solanacearum, however the transiently overexpressed $N$. benthamiana was more sensitive to the tobacco fungal pathogen Fusarium solani var. coeruleum. These results provide a reference for further research on the resistance function of sugarcane WRKY genes.

In the process of growth and development, plants often suffer from various external environmental challenges, such as drought, high salinity, cold, and pathogen stresses. After receiving stress signals in adversity, a complex regulatory network is formed in plants. Through the expression changes of various stress-related functional genes, the transcripts are recombined in plants to enhance adaptability to the environment. These gene expression changes and modifications are mainly regulated by transcription factors ${ }^{1,2}$. At present, several plant stress-related transcription factors have been reported, such as WRKY $[\operatorname{Trp}(\mathrm{W})-\operatorname{Arg}(\mathrm{R})-\mathrm{Lys}(\mathrm{K})-\operatorname{Try}(\mathrm{Y})$, tryptophan-argininelysine-tyrosine $]^{3}$, MYB (myeloblastosis) ${ }^{4}$, NAC [also call as NAM, ATAF1(2), CUC2] ${ }^{5}$, bZIP (basic leucinezipper $)^{6}$, AP2/ERF (APETALA 2/ethylene-responsive element binding factor) ${ }^{7}, \mathrm{C} 2 \mathrm{H} 2$ zinc finger protein ${ }^{8}, \mathrm{SPL}$ $\left(S Q U A M O S A \text { promoter-binding protein-like) }{ }^{9} \text {, DREB (dehydration responsive element binding protein) }\right)^{10}$, and CDFs (cycling DOF factors) ${ }^{11}$.

WRKY is considered to be a plant-specific transcriptional regulator ${ }^{12}$. Since the first sweet potato WRKY gene SPF1 (SWEET POTATO FACTORS1) was cloned in $1994^{13}$, a large number of WRKY family genes have been gradually isolated and identified from various plants. There are 72 WRKY genes in the model plant Arabidopsis thaliana $^{3}, 105$ WRKYs in Oryza sativa ${ }^{14}, 68$ WRKYs in Sorghum bicolor ${ }^{15}, 45$ WRKYs in Hordeum vulgare ${ }^{16}, 119$ WRKYs in Zea mays ${ }^{17}$, and 105 WRKYs in Setaria italica ${ }^{18}$. The most typical feature of WRKY transcription factors is that there is a polypeptide sequence of at least 60 amino acids in length in the DNA-binding domain of WRKY family members. A highly conserved heptapeptide sequence WRKYGQK occurs near the N-terminus of WRKY, which is an important criterion for identifying the WRKY family members ${ }^{12}$. A zinc-finger-like motif is found in the WRKY domin ${ }^{12}$. Based on the number of WRKY domains and the characteristics of the zinc-fingerlike motif, the WRKY family can be divided into three types. Type I WRKYs contain two WRKY domains and a zinc-finger-like motif $\mathrm{C}_{2} \mathrm{H}_{2}\left(\mathrm{CX}_{4-5} \mathrm{CX}_{22-23} \mathrm{HX}_{1} \mathrm{H}\right)$ (X represents any amino acid residue). The WRKY domain

\footnotetext{
${ }^{1}$ Key Laboratory of Sugarcane Biology and Genetic Breeding, Ministry of Agriculture, College of Agriculture, Fujian Agriculture and Forestry University, Fuzhou 350002, Fujian, China. ${ }^{2}$ College of Life Sciences, Fujian Agriculture and Forestry University, Fuzhou 350002, Fujian, China. ${ }^{3}$ Key Laboratory of Genetics, Breeding and Multiple Utilization of Crops, Ministry of Education, College of Agriculture, Fujian Agriculture and Forestry University, Fuzhou 350002, Fujian, China. ${ }^{\boxplus}$ email: queyouxiong@126.com; syc2009mail@163.com
} 
A ScWrky 5 SC-WRKY SCWRKY 3 SCWRKY 4 SCWRKY 6 Consensus

SCWRKY 5 SC-WRKY SCWRKY 3 SCWRKY 4 SCWRKY 6 Consensus

SCWRKY 5 SC-WRKY SCWRKY 3 SCWRKY 4 SCWRKY 6 Consensus

SCWRKY 5 SC-WRKY SCWRKY 3 SCWRKY 4 SCWRKY 6 Consensus

SCWRKY 5

SC-WRKY

SCWRKY 3

SCWRKY 4

SCWRKY 6

Consensus

SCWRKY 5

SC-WRKY

SCWRKY 3

SCWRKY 4

SCWRKY 6

B Consensus

SCWRKY 5

SbWRKY 32

ZmWRKY 22

SIWRKY29

OSWRKY 64

TUWRKY 38

BdWRKY 38

Consensus

SCWRKY 5

SbWRKY 32

ZmWRKY 22

SIWRKY29

OSWRKY 64

TUWRKY 38

BdWRKY 38

Consensus

SCWRKY 5

SbWRKY 32

ZmWRKY 22

SIWRKY 29

OSWRKY 64

TUWRKY 38

BdWRKY 38

Consensus

SCWRKY 5

SbWRKY 32

ZmWRKY 22

SIWRKY29

OSWRKY 64

TUWRKY 38

BdWRKY 38

Consensus

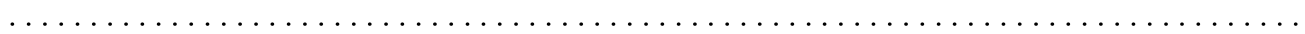
.

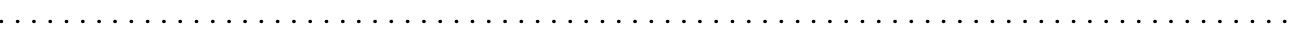

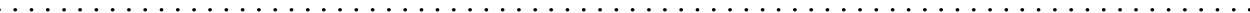
MEEVEEANREAVQSCHRVLALLSQPHDPAQVRS IALGTDEACAKFRKVVSLLSNGGVGVGEAGPSDASGSASHPRAKLVS (1)

$\ldots \ldots \ldots \ldots \ldots \ldots$ MAKRDDYMDSSRGCSNGTPKRLLQDCSSY . . . AQAHAKKKVRISTRTEYTYAPYHD $\ldots \ldots \ldots \ldots \ldots \ldots$ MAASLG LAHEACYAAYPPAAAASSYFPS . . . . . PPP PPGDLVAEFPPPAAATAMV $\ldots \ldots \ldots \ldots \ldots \ldots \ldots$ MQAYMEGGQLSACLPSFLVPDHYAGFPLPL . . PLQLPSQPNNKLFQMPFVVNQEETE $\ldots \ldots \ldots \ldots \ldots \ldots$ MEGSSQ . LLETCLPASLYALS PCAPPHP . . . PLLAPLPNQHKLLQMPLVQEP. AAN RRQNPGFLTQKGFLDTNTSVVVLNSAHPSPTSAQVYPRTAVALDAQGVHPLGGP PKLVQPLSAHFQFGNVPS PYQFPNQQ

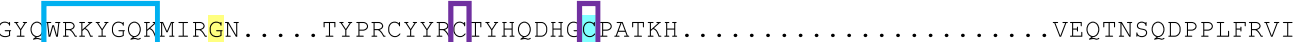

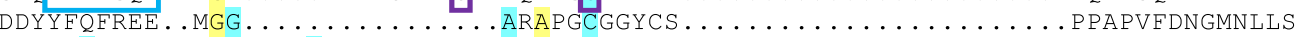
NHGGMLSSDH . CGG. LYPLPALPFGSCSGAAAATACGGKPT . . . . . . . AGFM . . . . . . PSAIGAEEVCTSVATKLG NHGVMLFSDHHQHGGPLYPLLLPG I PFCPSAAPATCEKTTTTGFTALDAGEAGRT . . . . . . SAAKAAAE IASTTTTTCN QQQQKLQAEMFKRSNS G INLKFEST T GTGTMS SARS F LS S LSKD GSVAS LDGKS S SFHLI GGPAMS DPVNAQQAPRRRCT

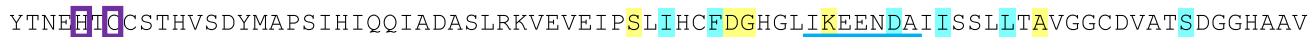
YGSADGDGRRLMS GPAGGNGGGRPASLIG .....FRTRSEVDVLDDGFKWRKYGKKAVKSS PNPRNYYF ISAEG . GGVKK CNESNSTWWKGSAATTAERGKMKVRRKMREPRFCFQTRS DVDVLDDGYKWRKYGQK VVKNS LHPRSYFP THSN. dRVKK G. PS SCNWWKG. PAAAGEKGRMKVRRKMRE PRFCFQTRS DVDVLDDGYKWRKYGQK VVKNS LHPRSYYR TTHSN. CRVKK GRGEDGTGKCTVTGRCHCSKRSRKLRVKRS I KVPA I SNK I ADI P P DECS WRKYGQK P IKGS PHPRGYYK SSVRCdPARK

QEETPARMSRGSNAASPSISPVLLPASDNLKTDFMEQLEPQWFEPLDLGWFIE $\ldots \ldots \ldots \ldots \ldots \ldots \ldots \ldots$ RVERDRDDPRYVITTYDGVFN FAAPGAAYVCP PPRGASTTPCFSPPYSASSAPLVAAPSWNAAF DAWKAQLHAAAAHSSE

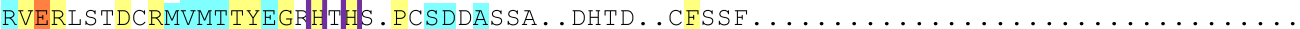
RVERLSEDCRMVITTYEGRHTHS. PCSDDADAAAGDHTGSCAFTSL $\ldots \ldots \ldots \ldots \ldots \ldots \ldots \ldots \ldots \ldots \ldots$

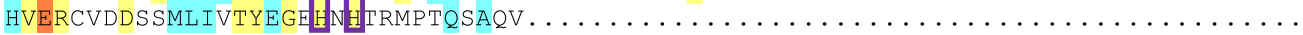
e

$\dot{s i s}$

SS

MAKRDDYMDSSRGCSNG. TPKRLLODCSS. . YAOAHAKKKVRISTRTEYTYAPYHDGYCWRKYGOKMIRGNTYPRCYYRC MAKRDDYMDSSCGCSNG. TPKRLVQDCSS. . YAQAHAKKKVRISTRTEYTYAPYHDGYCWRKYGQKMIRGNAYPRCYYRC MAKRGDYMDSSCDCSNGRAPKRLVQDCSS. . YAQAHAKKKVRISTRTEYTYAPYHDGY CWRKYGQKMIRGSTYPRCYYRC ........MAGFSGSSNG. THKRLLQDCSS. .YAQEHAKKRVRI STRTEYTYAPYHDGYCWRKYGQKMI RGNTYPRCYYFC MVKRS DNMDS S SECSRG. AHKRLLQDSRS. . Y DQENAMKKVC I GTRTEYTYAPYHDGY $ৎ$ WRKYGQKMIRGNSFPRCYYFC ......MLSS SGCPSGSNKRT $L Q D C S G$ GS HAQEHTKKKSRI GMRTDYTYAPYHDG FC WRKYGQKVIRGNAFPRCYYRC . MAKSGGSSSSGS DGANKRPLQDSVGG. . CAQEHAKKKTRVGVRTDYTYAPYHDGFCWRKYGQKMIRGNIFPRCYYPC

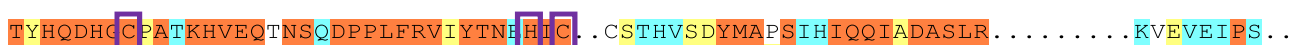

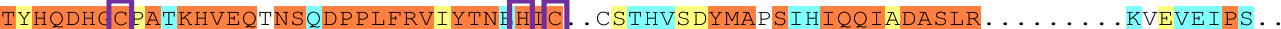
TYHQDHC C PATKHVEQTNSQDPPLFRVIYTNH H TYHQDH C CATKHVEQNNSQDPPLFRVIYTNH H I C. . CSTHVSDYMASSIHIQQIVDASLR . . . . . . EAEVEIPSQ. TFHQDHC C PATKHVEQTNSQDP PLFRVIYTNH H IC. . SSTHVS DYMASS IHIQQIADASLR . . . . . . . KAETEIPS. TYHQDHC C PASKHVEQHNSEDP L LRVIYTNH H C. TSNSAS DYMAS SMQIQQIADAS LRKAQAAERLRKAEVETPRLM TYHQDHC CSASKHVEQHNSADP PLFRVVYTN I H I C GAAATAS DYMAS SMH I QQ IADAS LRKADT . . . . EAE . RPPRPQ TYHQDHC CPASKHVEQSNSEDPPLFRVIYTNLH H C FGASEQYC. YMASSMQIQQIADASLRKPAP ...... ETTPAPPLPQ $t$ hqdhgc a khveq ns dpplfrv ytn htc yma s iqqi daslr

$\mathrm{P}$

$\ldots .$. LIHCFDGHGL . IKEENDAIISSLLTAVGG . CDVATSDGG . HAAVQ . . . . . . . EETP. . ARMSRG. SNAASPS .... ITHCFDGHGL. IKEENDAIISS. LTAISD . . YDVATSDGG . HAAVQ . . . . . . . EDTP . . ARMSRS. SNEASPS

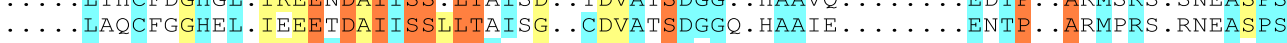
..... LTHCGAGHGL. IKEEKDAIVSSLLTVTNG . . CDVAKSDVG. . RAAMQ . . . . . . ENTP. . APMARN. IYEAIPS HS . . PPPRCSGGYNMAMKEEKDVIVSSLLTVIRG . . CHIAESAGNNSAAALP . . . . . . . VNRPP PAVARSD. HYSCSYA OP .... RSGGGGYAAAI KEEKDAIVSSLLTVIRG . SCDVVKSDTAHEGYSSASLASNCYAMS SPSVAGGSR . .EGSSSSS QQQLARRGRAAAYAAAIKQEKDAIVSSLLTVIRGCEVDVVKSEPVHENYGAG . . . . . ACMLSPPVAGGASGLEGSGSSL
$e \mathrm{~d}$ s. $1 t$

ISPVLLPAS. DNLKT . . DFMEQ . . LEPQWFEPLDLGWFIE . . . . . .

ISPVLLPAS. DNLKT . . DFIEQ . . . LEPQWFEPLDLGWFI. . . . ISPVLLRASSDKLKT . . DFIEP . . . LETQWFELLDLGWFIE . . . . VSPVQLAPS. DELKM . DFVEP . . . LESHWFEPLDLGWFI . . . . . ISPELLPAS. DDLTL. . DFMLDS. VLDPHWVEPLDLAWLKESTHT VSPVVLPAP. DDMGLGLDFMVES . . . . HWFEPLDLGWFVE . . . $\mathrm{sP}$
$\mathrm{VSPVAVPAP}$ $d f$ $w$ e ldl w 
4Figure 1. Protein sequence alignment of ScWRKY5 and WRKYs from sugarcane and other plant species by DNAMAN (version 6.0.3.99, Lynnon Biosoft) software. (A) The amino acid sequences of Sc-WRKY (ACT53875.1), ScWRKY3 (AYH64982.1), ScWRKY4 (AUV50355.1), ScWRKY6 (AXY87953.1) were identified from sugarcane. (B) The amino acid sequences of Sorghum bicolor SbWRKY32 (XP_002456546.1), Zea mays ZmWRKY22 (NP_001147816.1), Setaria italica SiWRKY29 (XP_004972233.1), Oryza sativa OsWRKY64 (XP_015642042.1), Triticum urartu TuWRKY38 (EMS45972.1), and Brachypodium distachyon BdWRKY38 (XP_010233534.1) were obtained from GenBank. The orange, yellow, blue, and white colors indicate the homology level of the conservation of the amino acid residues in the alignment at $100, \geq 75, \geq 50$, and $<50 \%$, respectively. The sequences of the WRKY motif (WRKYGQK or WRKYGKK) and the $\mathrm{C}_{2} \mathrm{HC}$ domain $\left(\mathrm{CX}_{7} \mathrm{CX}_{24} \mathrm{HX}_{1} \mathrm{C}\right)$ or $\mathrm{C}_{2} \mathrm{H}_{2}$ domain $\left(\mathrm{CX}_{5} \mathrm{CX}_{23} \mathrm{HX}_{1} \mathrm{H}\right)$ are highlighted by the blue and purple rectangles, respectively.

at the C-terminus of type I WRKYs mainly mediates the binding of the protein to the target DNA, while the function of the N-terminal WRKY domain remains to be studied. Type II WRKYs contain only one WRKY domain, and their zinc-finger-like motif is the same as the type I WRKYs. According to the homology between family members, type II WRKYs can be further divided into five subclasses (IIa-IIe) ${ }^{12}$, while, Dong et al. ${ }^{19}$ divided the type II WRKYs of $A$. thaliana into seven subclasses (IIa-IIg). Only one WRKY domain is contained in type III WRKYs, and the zinc-finger-like motif $\mathrm{C}_{2} \mathrm{HC}\left(\mathrm{CX}_{7} \mathrm{CX}_{23} \mathrm{HX}_{1} \mathrm{C}\right)$ differs from that of the type I and type II WRKYs ${ }^{20}$. Based on amino acid sequence similarity, $97 \mathrm{WRKY}$ proteins in $O$. sativa were divided into three types and 13 groups, of which class II WRKYs were divided into 10 subclasses (IIa-IIj), and class III WRKYs were divided into two subclasses (IIIa and IIIb) ${ }^{21}$.

WRKY transcription factors play an important role in the response to biotic and abiotic stresses. In 1996, Rushton et al..$^{22}$ found that WRKY1, WRKY2, and WRKY3 in Petroselinum crispum have regulatory roles in the PR1 gene-mediated immune response. Forty-nine of the $72 W R K Y$ genes in A. thaliana were induced by Pseudomonas syringae and salicylic acid (SA) stresses ${ }^{19}$. However, the expression profile of WRKYs in H. vulgare, namely, $H v W R K Y 1$ and $H v W R K Y 2$, was found to negatively regulate the resistance of $H$. vulgare to powdery mildew $^{23}$. In O. sativa, 15 WRKYs were induced by Magnaporthe grisea infection, of which 12 could also be simultaneously induced by Xanthomonas oryzae pv. oryzae $e^{24}$. There were 88 WRKYs in Phaseolus vulgaris response to drought stress, among which eight were up-regulated and 11 were down-regulated ${ }^{25}$. Transcriptome analysis showed that of the 100 WRKYs in Populus, 61 were induced by Marssonina brunnea, SA, methyl jasmonate (MeJA), injury, cold, and salt stresses ${ }^{26}$. In maize, 58 WRKYs were induced by drought stress ${ }^{27}$.

Sugarcane (Saccharum spp.) is an important sugar crop globally ${ }^{28,29}$. Liu et al..$^{30}$ found that the expression level of the class II sugarcane $S c$-WRKY gene (GenBank Accession No. GQ246458) was induced by the sugarcane smut pathogen (Sporisorium scitamineum), SA, sodium chloride ( $\mathrm{NaCl}$ ), and polyethylene glycol (PEG), suggesting that the $S c$-WRKY gene may play a role in the response mechanism of sugarcane to $S$. scitamineum, drought, and high salt stresses. Wang et al. ${ }^{31}$ showed that the expression level of class IIc sugarcane ScWRKY3 gene (GenBank Accession No. MK034706) was decreased in the smut-susceptible Saccharum hybrid variety ROC22 and remained unchanged in the smut-resistant Saccharum hybrid variety Yacheng05-179. Additionally, the expression level of $S c W R K Y 3$ was increased by $\mathrm{NaCl}, \mathrm{PEG}$, and the plant hormone abscisic acid (ABA), but was decreased by SA and MeJA. In contrast, Wang et al. ${ }^{32}$ indicated that the expression level of class IIc sugarcane ScWRKY4 gene (GenBank Accession No. MG852087) was repressed in the Yacheng05-179 and remained unchanged in ROC22 after inoculation with $S$. scitamineum. The expression level of ScWRKY4 gene was increased under ABA, $\mathrm{SA}, \mathrm{MeJA}, \mathrm{NaCl}$, and PEG stresses. Zhang et at. ${ }^{33}$ found that the expression level of class IId sugarcane ScWRKY6 gene (GenBank Accession No. MH393927) was increased under NaCl, PEG, and MeJA stresses. Li et al. ${ }^{34}$ showed that the expression profile of SsWRKY in different Saccharum spontaneum samples at 46 different developmental stages had different spatial and temporal patterns, with 52 SsWRKY genes expressed in all of the tissues, four SsWRKY genes not expressed in any tissues, and 21 SsWRKY genes possibly involved in photosynthesis. These above researches indicate that sugarcane WRKY family members of different types may have various functions due to their expression characteristics. In this study, a new class III WRKY gene, ScWRKY5, was successfully cloned from sugarcane variety ROC22. The sequence characteristics, subcellular localization, transcriptional self-activation activity, tissue-specific expression, gene expression patterns under different stresses, and transient expression of ScWRKY5 in Nicotiana benthamiana after inoculation with the tobacco pathogens Ralstonia solanacearum and Fusarium solani var. coeruleum were analyzed.

\section{Results}

Cloning and sequence analysis of the ScWRKY5 gene. The cDNA sequence of the ScWRKY5 gene (GenBank Accession No. MK629767) with a total length of 1359 bp was successfully obtained from the mature leaves of the sugarcane variety ROC22. The ScWRKY5 protein encodes 238 amino acid residues and has a theoretical molecular weight of $26.78 \mathrm{kDa}$. The isoelectric point, average hydrophobicity, and instability coefficient of the ScWRKY5 protein were 6.11, - 0.611, and 56.46, respectively, suggesting that ScWRKY5 is an acidic, unstable, and hydrophilic protein. Amino acid sequence alignment indicated that the ScWRKY5 protein has low homology with previously reported class II sugarcane WRKYs (Sc-WRKY, ScWRKY3, ScWRKY4, and ScWRKY6), which was $7.66 \%, 8.98 \%, 10.08 \%$, and $8.76 \%$, respectively (Fig. 1A). The amino-acid sequence similarity of the ScWRKY5 protein to S. bicolor SbWRKY32 (XP_002456546.1), Z. mays ZmWRKY22 (NP_001147816.1), S. italica SiWRKY29 (XP_004972233.1), O. sativa OsWRKY64 (XP_015642042.1), Triticum urartu TuWRKY38 (EMS45972.1), and Brachypodium distachyon BdWRKY38 (XP_010233534.1) was 94.09\%, 85.12\%, 81.30\%, $61.54 \%, 57.53 \%$, and 54.66\%, respectively (Fig. 1B). A conserved WRKY domain (WRKYGQK) and a zinc-finger-like motif $\left(\mathrm{CX}_{7} \mathrm{CX}_{24} \mathrm{HX}_{1} \mathrm{C}\right)$ were found at the $\mathrm{C}$-terminus of these seven WRKY proteins (Fig. 1B), whereas 


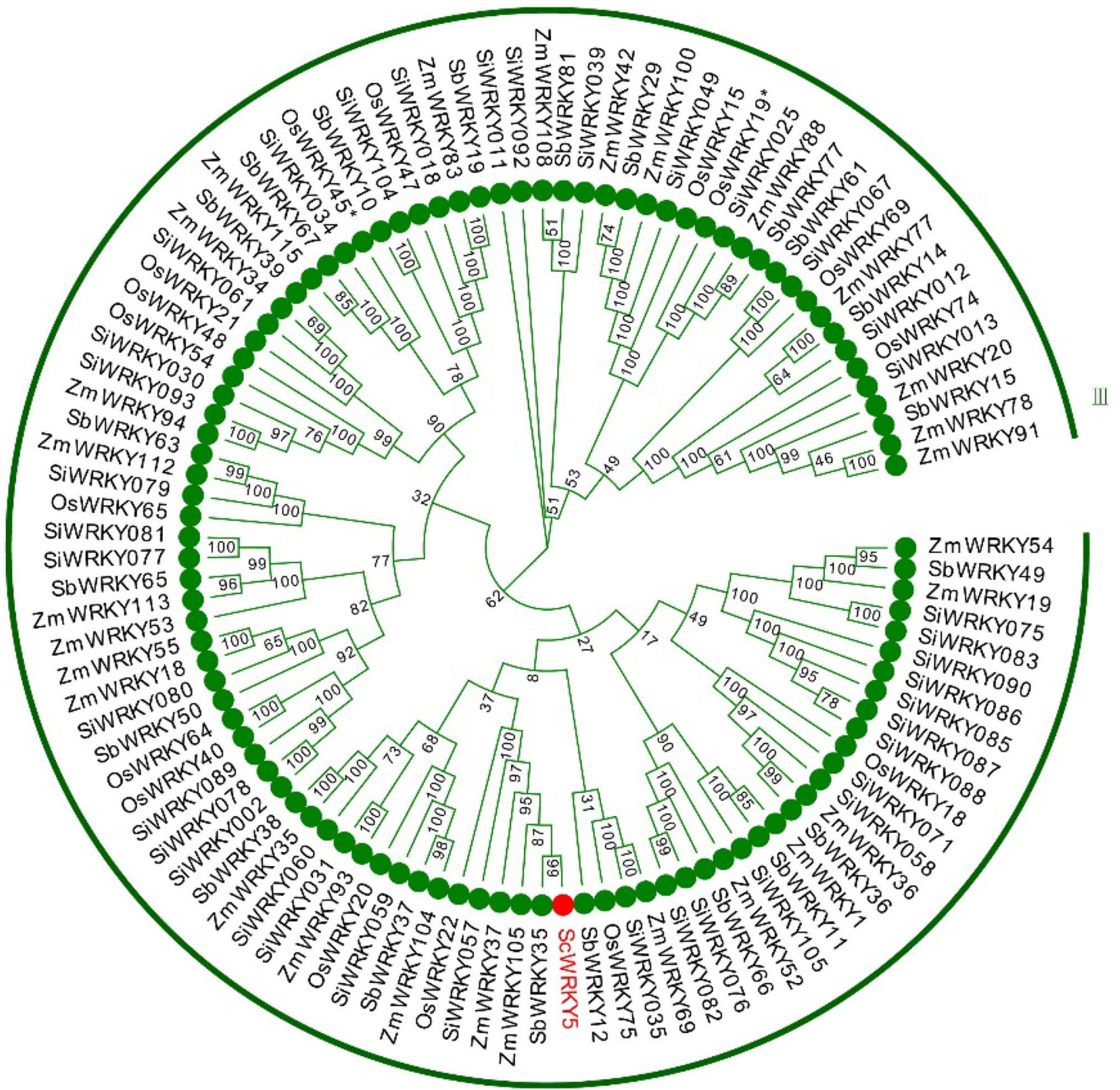

Figure 2. Phylogenetic tree analysis of the ScWRKY5 protein and WRKYs from other plant species. The phylogenetic tree was plotted using the neighbor-joining (NJ) method with 1000 bootstrap replicates in MEGA 7.0 software. The ScWRKY5 protein is marked by a red circle. ZmWRKY, SbWRKY, SiWRKY, and OsWRKY represent the WRKYs from Zea mays, Sorghum bicolor, Setaria italica, and Oryza sativa, respectively.

a WRKY domain (WRKYGKK or WRKYGQK) and a zinc-finger-like motif $\left(\mathrm{CX}_{7} \mathrm{CX}_{24} \mathrm{HX}_{1} \mathrm{C}\right.$ or $\left.\mathrm{CX}_{5} \mathrm{CX}_{23} \mathrm{HX}_{1} \mathrm{H}\right)$ were contained at the four reported class II sugarcane WRKYs (Fig. 1A). Phylogenetic tree analysis showed that ScWRKY5 and the WRKY family proteins from S. spontaneum could be divided into three categories (I-III) (Fig. S1). The ScWRKY5 protein was classified into group III and showed $93.99 \%$ and $93.70 \%$ amino acid sequence similarity with the S. spontaneum WRKY (Sspon.03g0003780-2c) and S. bicolor SbWRKY35 (Sb03g038170) (Figs. S1 and 2). The cis-acting elements contained in the promoter of S. spontaneum WRKY (Sspon.03g0003780-2c) were predicted online using the PlantCARE tool. The upstream 2000 bp promoter of the S. spontaneum WRKY (Sspon.03g0003780-2c) contained 20 types of cis-acting elements (Table S1), including a large number of the core promoter elements TATA-box (54 sites) and CAAT-box (21 sites), as well as many other types of cis-acting elements. There were eight types of light-response elements, which were the cis-acting elements of the GA-motif, GATA-motif, 4 cl-CMA2b, GT1-motif, G-Box, G-box, box 4, and AE-box. Several response hormone signal substances were predicted, such as the TGACG-motif, P-box, ABRE, and TCA-element, which may participate in MeJA, gibberellin (GA), SA, and ABA reactions, respectively. There were also some elements related to the abiotic stress response, such as MBS cis-acting elements involved in drought stress. 

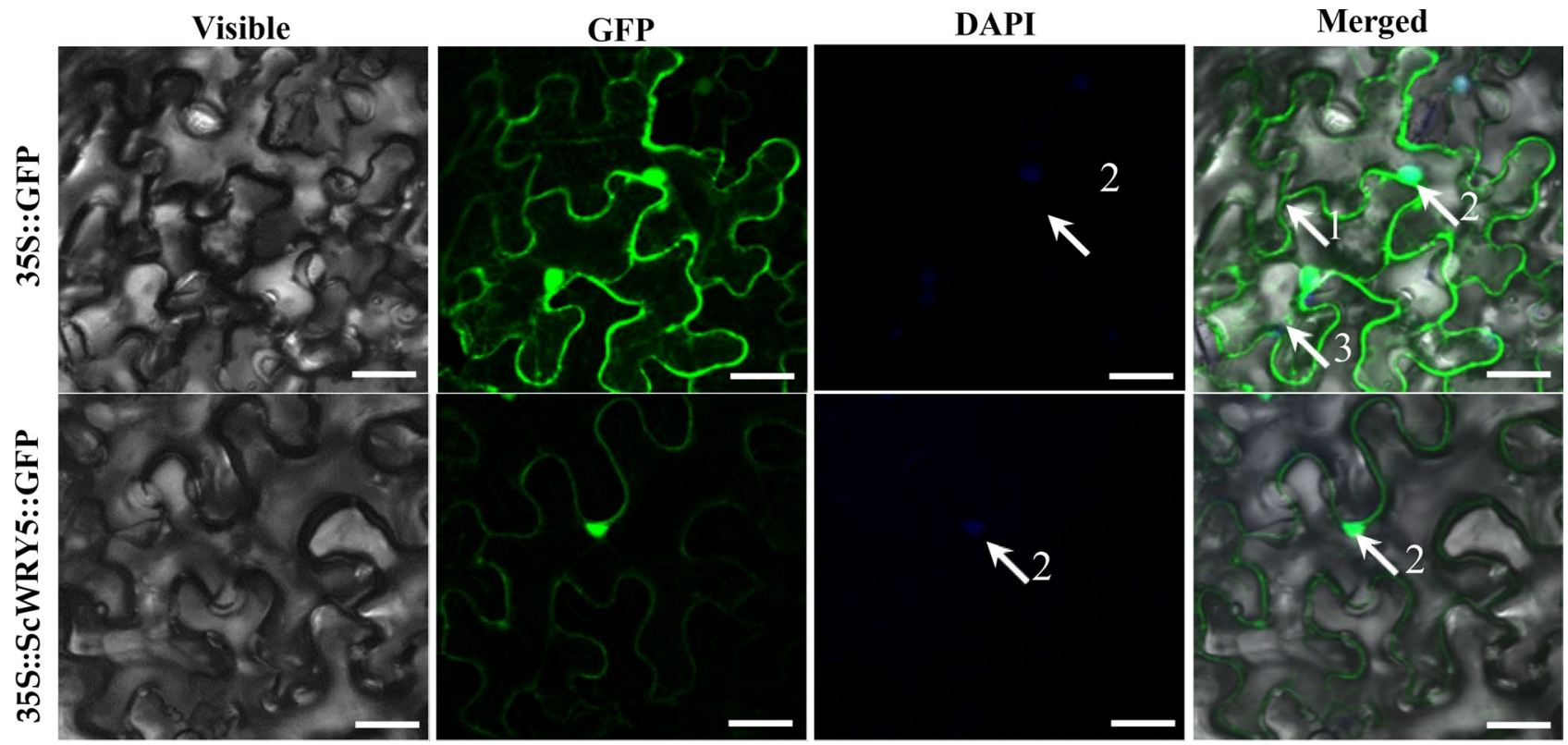

Figure 3. Subcellular localization of ScWRKY5 in Nicotiana benthamiana leaves. The epidermal cells of $N$. benthamiana were used for capturing images of visible light, green fluorescence, blue fluorescence, and visible light merged with green and blue fluorescence. White arrows 1, 2, and 3 indicate the cell membrane, nucleus, and cytoplasm, respectively. Scale bar $=50 \mu \mathrm{m}$. 35S::GFP, the Agrobacterium tumefaciens strain carrying the empty vector pMDC83-GFP. 35S::ScWRKY5::GFP, the A. tumefaciens strain carrying the recombinant vector pMDC83-ScWRKY5-GFP. GFP, green fluorescent protein; DAPI, 4',6-diamidino-2-phenylindole.

The promoter element and functional prediction indicated that $S c W R K Y 5$, the homologous gene of the S. spontaneum WRKY (Sspon.03g0003780-2c), may be regulated by photosystem, hormone signaling substances, and abiotic stresses.

Subcellular localization of ScWRKY5. The Euk-mPLoc 2.0 Server predicted the subcellular localization of the sugarcane ScWRKY5 protein in the nucleus. However, the WoLF PSORT predicted the subcellular localization of the ScWRKY5 protein in the nucleus with the highest probability, followed by in the chloroplast, cytoplasm, and peroxisome. Here, to validate the true location of the ScWRKY5 protein, the fusion expression plasmid pMDC83-ScWRKY5-GFP was constructed, and the subcellular localization expression vector pMDC83-GFP was used as a positive control. Using Agrobacterium-mediated transformation and injecting into $N$. benthamiana leaves, the ScWRKY5-GFP fusion protein emitted green fluorescence predominantly in the nucleus, whereas the fluorescence of free GFP was detected in almost around all cell space (Fig. 3). These results indicated that the ScWRKY5 protein is localized in the nucleus.

Transcriptional activation activity of ScWRKY5. The Y2HGold-GAL4 yeast two-hybrid system was used to analyze the self-activating activity of the target protein. On the SDO (synthetic dropout medium without tryptophan) plate, the positive control pGADT7 + pGBKT7-p53 and the negative controls pGBKT7 and pGBKT7-ScWRKY5 could grow normally, indicating that the target plasmid pGBKT7-ScWRKY5 had been successfully transferred into the yeast Y2HGold, and tryptophan could be successfully expressed in GAL4-BD combined with the ScWRKY5 protein (Figs. 4 and S2). On the SDO plate with X- $\alpha$-Gal dye added, the positive control and the yeast cells transformed with pGBKT7-ScWRKY5 all turned blue, suggesting that the MEL1 gene had been activated. Further tests showed that the positive control grew normally and turned blue on the SDO/X plate medium with the addition of the aureobasidin A (AbA), indicating that the reporter genes $A U R 1-C$ and MEL1 had been activated. The blue colonies of the yeast cells transformed with pGBKT7-ScWRKY5 proved that the protein of ScWRKY5 exhibited transcriptional activation activity.

Tissue-specific expression of ScWRKY5 in sugarcane. The relative expression level of the ScWRKY5 gene in the sugarcane root, bud, leaf, stem pith, and stem epidermis was detected by quantitative real-time PCR (qRT-PCR) (Fig. 5). The results indicated that this gene was constitutively expressed in all of these tissues. The lowest expression level of $S c W R K Y 5$ occurred in the root, while its relative expression level in the stem pith and leaf was 6.61 -fold and 4.45 -fold higher than that in the root, respectively.

Expression patterns of SCWRKY5 under different stresses. The qRT-PCR method was used to analyze the expression patterns of the ScWRKY5 gene under different exogenous stresses (Fig. 6). Under $5 \mathrm{mM} \mathrm{SA}$ stress for $3 \mathrm{~h}$, the gene expression level of ScWRKY5 was 3.74-fold higher than that of the control (Fig. 6A). Within $24 \mathrm{~h}$ of $100 \mu \mathrm{M}$ MeJA treatment, the gene expression level of ScWRKY5 was relatively stable (Fig. 6A). 


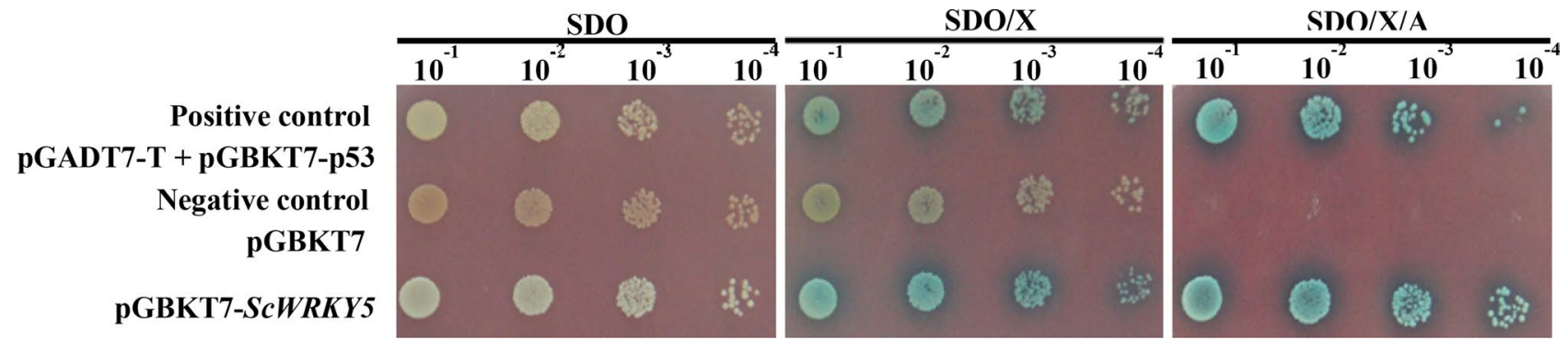

Figure 4. The ScWRKY5 transactivation activity test. SDO (SD/-Trp), synthetic dropout medium without tryptophan; SDO/X (SD/-Trp/X-a-Gal), synthetic dropout medium without tryptophan, but plus 5-bromo4-chloro-3-indoxyl- $\alpha$-D-galactopyranoside; SDO/X/A (SD/-Trp/X- $\alpha-G a l / A b A)$, synthetic dropout medium without tryptophan but plus 5-bromo-4-chloro-3-indoxyl- $\alpha-\mathrm{D}$-galactopyranoside and aureobasidin A. pGADT7-T + pGBKT7-p53, positive control; pGBKT7, negative control.

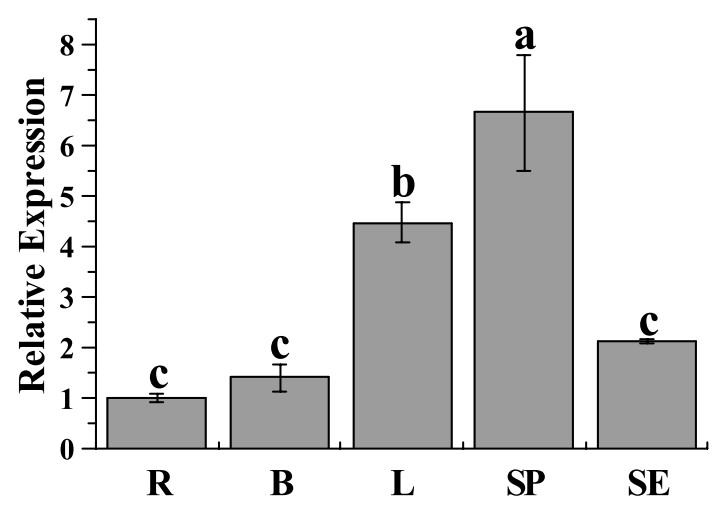

Figure 5. Tissue-specific expression of $S c W R K Y 5$ in different sugarcane tissues. The root, bud, leaf, stem pith, and stem epidermis tissues are represented by R, B, L, SP, and SE, respectively.

Under $100 \mu \mathrm{M}$ ABA stress, the gene expression level of ScWRKY5 was significantly increased at $3 \mathrm{~h}$, being 4.65fold higher than that of the control, following which it dropped to the control level at $24 \mathrm{~h}$ (Fig. 6A). Under $250 \mathrm{mM} \mathrm{NaCl}$ stress, the gene expression level of ScWRKY5 was stabilized from $0.5 \mathrm{~h}$ to $6 \mathrm{~h}$ and then rose rapidly to a peak at $24 \mathrm{~h}$, at which point it was 1.62 -fold higher than that of the control (Fig. $6 \mathrm{~B}$ ). Under $25 \%$ PEG 8000 stress, the transcription of ScWRKY5 remained unchanged at 0.5-6 h, but was increased to a maximum at $24 \mathrm{~h}$ which was 1.56 -fold higher than that of the control (Fig. 6B). In addition, the transcripts of ScWRKY5 was detected in the interaction between six different sugarcane varieties and S. scitamineum (Fig. 6C). In the smut-resistant varieties, the expression level of $S c W R K Y 5$ was significantly increased in YZ01-1413 and LC05136 after inoculation with the $S$. scitamineum for 1 day post-inoculation (dpi), which were 8.58- and 20.32-fold higher than that in the control group, respectively. However, the expression level of ScWRKY5 did not change significantly within $3 \mathrm{dpi}$ in the smut-resistant variety Yacheng05-179. Among the three smut-susceptible varieties, the expression level of ScWRKY5 was significantly decreased in YZ03-103 and FN40 from 1 to 3 dpi, while it was significantly decreased in ROC22 at $1 \mathrm{dpi}$ and returned to the control level at $3 \mathrm{dpi}$. These results revealed that the transcripts of ScWRKY5 could be induced by ABA, NaCl, PEG, SA, and S. scitamineum stresses.

Transient overexpression of ScWRKY5 in N. benthamiana leaves. The transient overexpression of A. tumefaciens cells carrying ScWRKY5 in N. benthamiana leaves was performed. The transcript of the ScWRKY5 gene was successfully detected in the $N$. benthamiana leaves using a semi-quantitative PCR technology (Figs. 7A and S3). The DAB (3,3'-diaminobenzidine) test showed that the staining color in the 35S::ScWRKY5 leaves was nearly the same as that of the control (Fig. 7B). The qRT-PCR results (Fig. 7C) indicated that in the 35S::ScWRKY5 leaves, the expression levels of the hypersensitive response (HR) marker gene NtHSR203, and JA pathway related genes NtPR2 and NtPR3 were all increased, which were 3.56-, 2.30-, and 7.00-fold higher than that of the control group, respectively. But the HR marker gene NtHSR515, the SA pathway related gene $N t P R-1 a / c$, and the ET synthesis-dependent genes NtEFE26 and NtAccdeaminase were all decreased, which were 0.20-, 0.13-, 0.14-, and 0.22 -fold lower than that of the control group, respectively. In addition, the expression level of HR marker gene NtHSR201 was relatively stable.

Phenotype analysis showed that there was no obvious symptom in the $35 S:: 00$ and $35 S:: S c W R K Y 5$ leaves at one-day after inoculation with $R$. solanacearum. However, the leaves were yellow and the allergic spots were more serious in the 35S::00 group after seven days (Fig. 8A). The qRT-PCR results indicated that the expression level of the NtPR2 gene was increased by the overexpression of the $S c W R K Y 5$ gene at one-day post inoculation, 

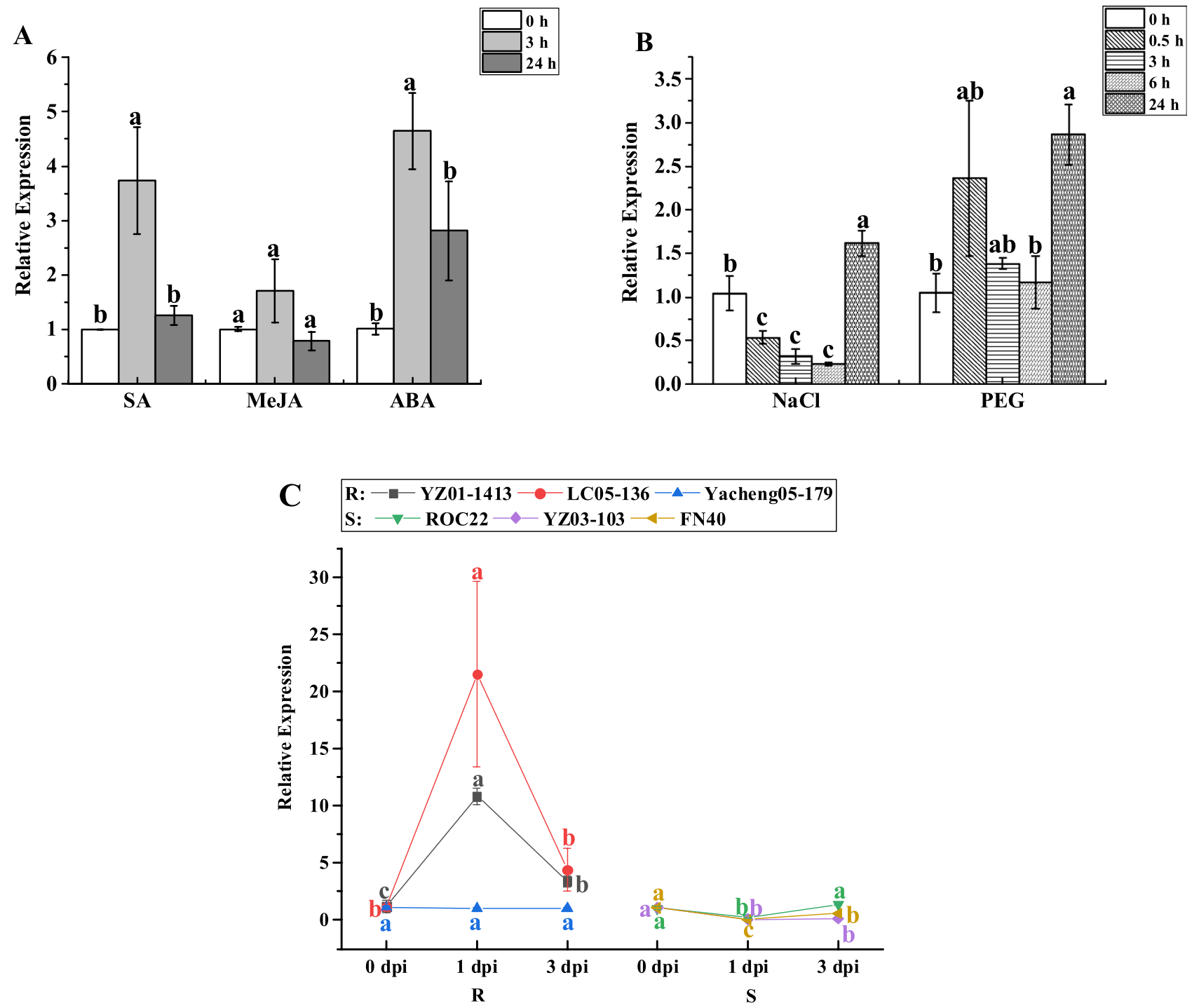

Figure 6. Expression patterns of $S c W R K Y 5$ under different stresses as determined by qRT-PCR. (A) Relative expression of ScWRKY5 in 4-month-old ROC22 plantlets under plant hormone stress. SA, salicylic acid (5 mM); MeJA, methyl jasmonate $(25 \mu \mathrm{M})$; ABA, abscisic acid $(100 \mu \mathrm{M})$. (B) Relative expression of ScWRKY5 in 4-month-old ROC22 plantlets under abiotic stress. NaCl, sodium chloride (simulating salt stress) (250 mM); PEG, polyethylene glycol (simulating drought treatment) (25\%). (C) Relative expression of ScWRKY5 in sugarcane after inoculation with Sporisorium scitamineum. YZ01-1413, LC05-136, and Yacheng05-179 were smut-resistant sugarcane varieties (R). ROC22, YZ03-103, and FN40 were smut-susceptible sugarcane varieties (S). dpi, days post-inoculation. Data are normalized to the glyceraldehyde-3-phosphate dehydrogenase $(G A P D H)$ expression level. All of the data points are means \pm standard error $(n=3)$. Bars superscripted by different lowercase letters indicate significant differences, as determined by Duncan's new multiple range test $(p$-value $<0.05)$.

which was 124.46-fold higher than that of the control group, and was repressed after seven days. The expression levels of the NtHSR201, NtHSR203, NtHSR515, NtPR-1a/c, NtPR3, and NtEF26 were repressed at one-day post inoculation, and recovered to the control level after seven days. The expression level of the NtAccdeaminase remained unchanged at one and seven days post inoculation (Fig. 8B).

The $N$. benthamiana leaves overexpressing the ScWRKY5 gene did not show symptoms after one day of inoculation with F. solani var. coeruleum. Conversely, the leaves turned yellow and wilted severely after seven days of inoculation, which was higher than that of the control group (Fig. 8C). The DAB results showed that as the infection time increased, the brown color of the tobacco darkened, and the leaves overexpressing the ScWRKY5 gene were darker than the control group after seven days. (Fig. 8C). The qRT-PCR showed that, compared with the control group, the expression level of NtHSR515 and NtPR2 was decreased in the 35S::ScWRKY5 leaves after inoculation for one day, and the expression level of $N t P R-1 a / c$ was stable at the control level. Instead, the expression levels of NtHSR201, NtHSR203, NtPR3, NtEFE26, and NtAccdeaminase were significantly increased in the $35 S:: S c W R K Y 5$ leaves, which were 1.55-, 2.54-, 22.17-, 1381.62-, and 3.55-fold higher than that of the control group, respectively. After seven days of infection, only the expression level of $N t P R-1 \mathrm{a} / \mathrm{c}$ was decreased, while 

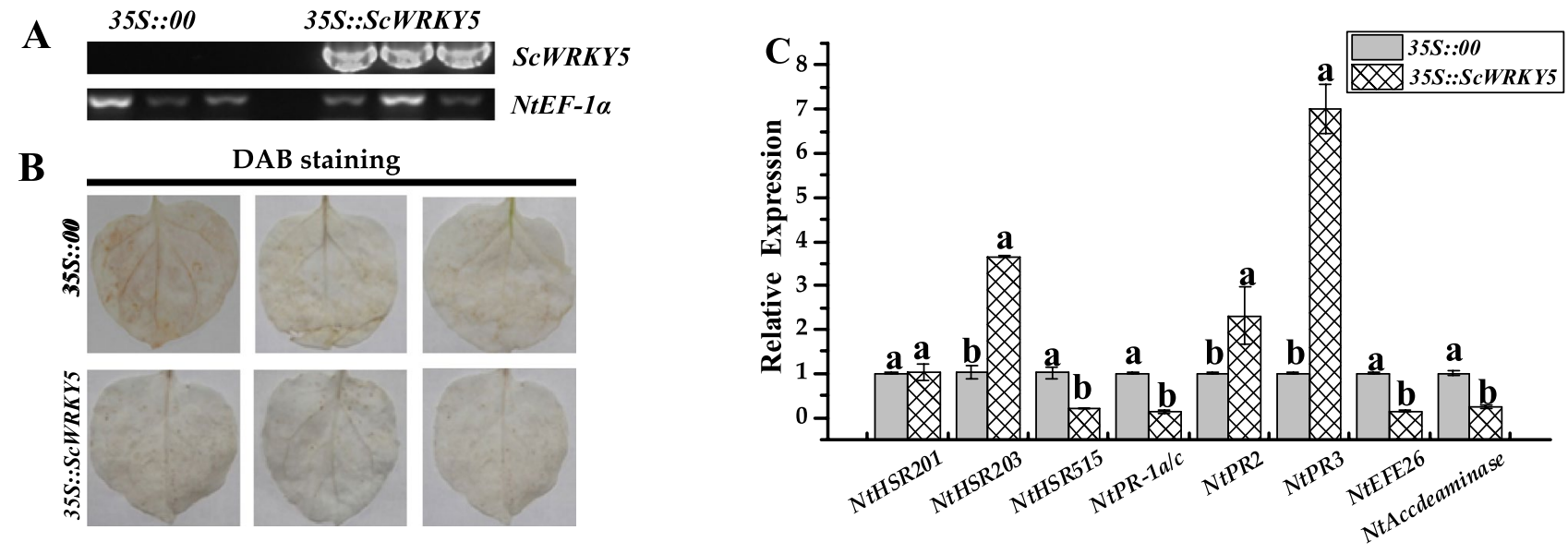

Figure 7. Transient overexpression of ScWRKY5 in Nicotiana benthamiana leaves. (A) Semi-quantitative PCR analysis of ScWRKY5 in N. benthamiana leaves inoculated with Agrobacterium GV3101 carrying pEarleyGate 203-ScWRKY5 (35S::ScWRKY5) and the empty vector pEarleyGate 203 (35S::00). (B) DAB staining analysis of $N$. benthamiana leaves after one day of inoculation. (C) Transcription levels of eight immune-related marker genes in $N$. benthamiana leaves after one day of inoculation. Data are normalized to the expression level of $N t E F-1 \alpha$. All data points are means \pm standard error $(n=3)$. Bars superscripted by different lowercase letters indicate significant differences, as determined by Duncan's new multiple range test $(p$-value $<0.05)$.

the expression of NtHSR515 and NtPR2 was stable at the control level, and the expression of the other five genes was still increased (Fig. 8D).

\section{Discussion}

WRKY is one of the largest transcription factors in plants and plays an important role in the plant response to biotic and abiotic stresses. As more WRKY transcription factors are being identified from different plants, their structural characteristics, origin, evolution, and gene functions are being increasingly studied ${ }^{3,18}$. However, there are only limited studies on WRKY genes in sugarcane ${ }^{30-34}$. In this study, the ScWRKY5 gene was isolated from sugarcane ROC22. The ScWRKY5 protein was clustered with the amino acid sequences from S. spontaneum in the class III WRKY family ${ }^{34}$ (Fig. S1). The physical and chemical properties of proteins are of great significance to their molecular biological functions. We predicted that the sugarcane ScWRKY5 was a hydrophilic protein with poor functional stability. Although ScWRKY5 belongs to the class III WRKY family, it only has one zincfinger-like motif $\mathrm{CX}_{7} \mathrm{CX}_{24} \mathrm{HX}_{1} \mathrm{C}$, which is the same as that of tomato SlWRKY80 ${ }^{35}$. As reported, only one of 81 tomato WRKY proteins has this zinc-finger-like motif $\left(\mathrm{CX}_{7} \mathrm{CX}_{24} \mathrm{HX}_{1} \mathrm{C}\right)$ variation ${ }^{35}$, suggesting that this type of structural variation in zinc finger is uncommon. Therefore, it is necessary to further assess whether the variant zinc structure of ScWRKY5 will cause the appearance or loss of some specific functions.

The location of proteins in cells is important for determining their biological functions ${ }^{36}$. The subcellular localization analysis of $164 \mathrm{NtWRKY}$ transcription factors in common tobacco showed that $118 \mathrm{NtWRKYs}$ (including most of the class I and class II WRKY family members) were located in the nucleus, and $77.4 \%$ of class III WRKYs were located in the cytoplasm ${ }^{37}$. In this study, the ScWRKY5-GFP fusion protein emitted green fluorescence predominantly in the nucleus (Fig. 3) which is consistent with the result predicted by the Euk-mPLoc 2.0 Server, suggesting that the ScWRKY5 protein may play the role of a nucleoprotein in sugarcane. However, for the small probability that ScWRKY5 protein was predicted to locate in the chloroplast, cytoplasm, or peroxisome by WoLF PSORT software, further subcellular localization test is needed in conjunction with promoter analysis of the ScWRKY5 gene itself as well as a western blot to check the integrity of the fusion protein in the tissues infiltrated.

Transcriptional activity is important for the functional analysis of transcription factors ${ }^{38}$. Since the GAL4 yeast two-hybrid system was firstly discovered ${ }^{39}$, this method has been increasingly used for studying the interactions between WRKY proteins ${ }^{40}$. In the present study, the protein encoded by the $S c W R K Y 5$ cDNA showed a self-activating activity (Fig. 4). Similarly, GhWRKY40 not only has a self-activating activity in yeast, but it also acts as a downstream interaction protein of GhMPK20, participating in plant-borne pathogenic signals through the MAPK cascade pathway ${ }^{41}$. Proteins with self-activation activity can be detected in sections to determine the specific location of the self-activation region of the protein, which can be verified in subsequent experiments.

The expression abundance of the WRKY gene in different plant tissues is not consistent ${ }^{42-44}$. There are 28 $C i W R K Y$ genes detected in the roots, stems and leaves of Caragana intermedia ${ }^{42}$. Different CiWRKY genes are expressed in different tissues, e.g., CiWRKY69-1 had the highest expression level in the roots, while CiWRKY40-1 and CiWRKY30 were mainly expressed in the leaves ${ }^{42}$. Bakshi et al. ${ }^{20}$ found that 12 of the 37 Arabidopsis WRKY genes were specifically expressed in the mature region of the root cells and may be involved in regulating the maturation of Arabidopsis root cells. TaWRKY44 was expressed differently in all of the tested organs, including the roots, stems, leaves, pistils, and stamens, with the highest expression level in the leaves and the lowest in the pistil $^{43}$. In this study, ScWRKY5 was expressed constitutively in the sugarcane tissues, with the highest expression 


\section{A}
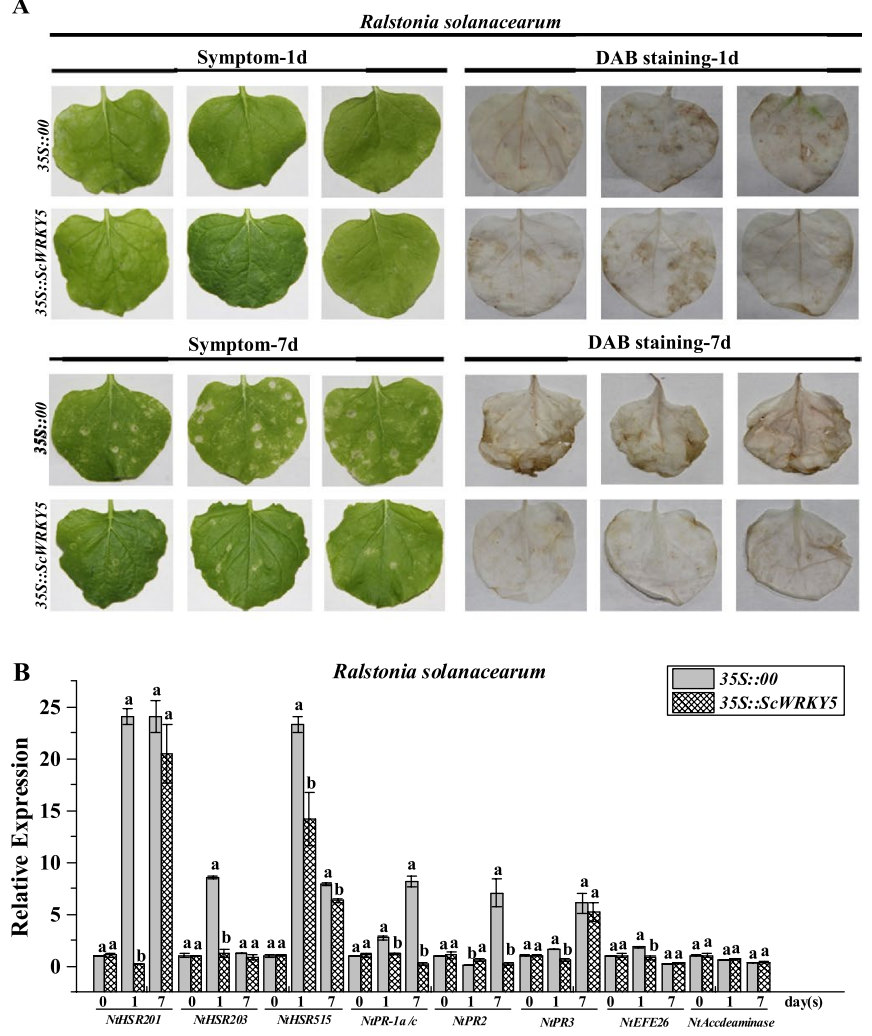

C
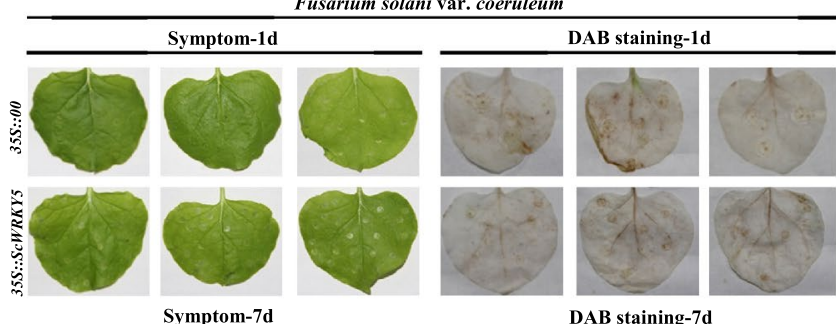

Symptom- $7 \mathrm{~d}$

DAB staining-7d
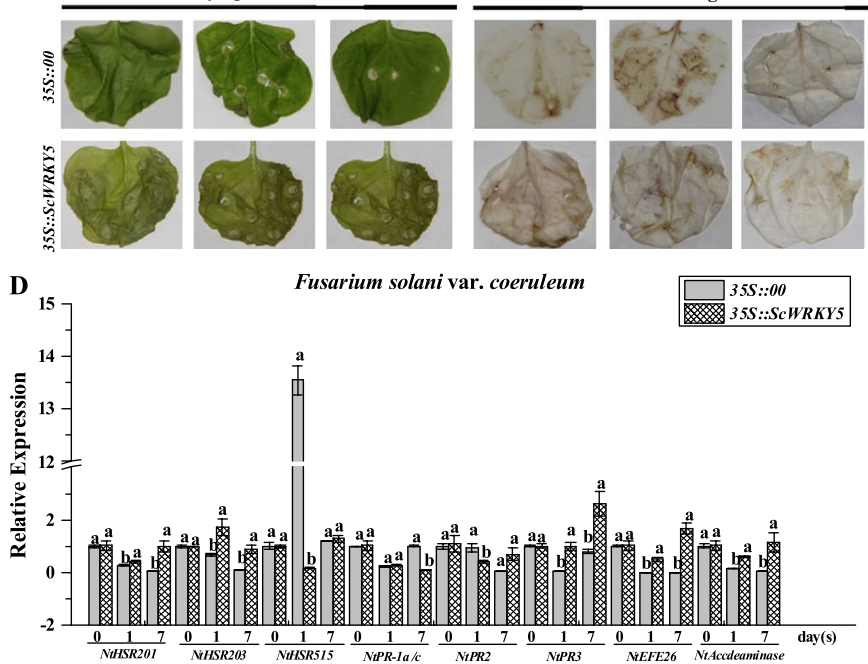

Figure 8. Resistance effect of the transient overexpression of ScWRKY5 in Nicotiana benthamiana leaves. (A,C) Phenotype and DAB staining analysis of $N$. benthamiana leaves inoculated with Ralstonia solanacearum and Fusarium solani var. coeruleum after one day and seven days of inoculation. (B,D) Transcription levels of eight immune-related marker genes in $N$. benthamiana leaves after one day and seven days of inoculation with $R$. solanacearum and F. solani var. coeruleum. Data are normalized to the expression level of NtEF-1 $\alpha$. All of the data points are means \pm standard error $(n=3)$. Bars superscripted by different lowercase letters indicate significant differences, as determined by Duncan's new multiple range test $(p$-value $<0.05)$. 
level in the stem pith, and the lowest level in the root (Fig. 5). These results indicated that WRKY genes may play different roles in the regulation of plant growth and development.

WRKY transcription factors can rapidly perceive signals of changes in external environmental conditions and transmit these signals in a timely manner in order for plants to respond to the environment ${ }^{12,45}$. Abiotic stresses, such as salt and drought can induce the expression of a series of stress response genes in the ABA signal transduction pathway, thereby enhancing the plant resistance ${ }^{46}$. Wang et $\mathrm{al}^{31}$ found that the expression level of the $S c W R K Y 3$ gene was increased under the stresses of $\mathrm{NaCl}, \mathrm{PEG}$, and the plant hormone ABA, but it was repressed under the stresses of SA and MeJA. In this study, the expression level of the ScWRKY5 gene was also increased by the stresses of PEG, NaCl, SA, and ABA (Fig. 6). It is speculated that the ScWRKY5 gene may play a role in the tolerance to salt and drought in sugarcane through SA and ABA signaling pathways. Similarly, under drought and salt conditions, the expression level of SIWRKY8 in Solanum lycopersicum is significantly increased, which occurs by directly activating the expression of SlWRKY8 in the SA and ABA synthesis pathways, thereby enhancing the resistance of $S$. lycopersicum to drought and salt stresses ${ }^{47}$.

Previous studies have shown that the GhWRKY40 gene is induced by the bacteria R. solanacearum upon infection and is up-regulated after treatment with SA, MeJA, and $\mathrm{ET}^{41}$. When GhWRKY40 was transiently overexpressed in tobacco leaves, most resistance-related genes, including SA, ET, JA, and HR response genes, were repressed after infection with $R$. solanacearum, suggesting that the overexpression of GhWRKY40 reduce the resistance to $R$. solanacearum ${ }^{41}$. Overexpressing AtWRKY28 and AtWRKY75 increased the tolerance to oxalic acid and the resistance to Sclerotinia sclerotiorum by participating in JA/ET-dependent defense signaling pathways in Arabidopsis ${ }^{48}$. The OsWRKY13 gene enhanced the defense ability of rice blast via cross-talk with the SA-dependent defense pathway, SNAC1-mediated abiotic stress defense pathway, and JA signaling pathway ${ }^{49}$. When infected with $R$. solanacearum, Arabidopsis WRKY27 has a negative regulatory effect on the expression of defense-related genes and exhibited earlier disease symptoms than the mutant WRKY27-1 plant, which had lost WRKY $27^{50}$. Overexpressing GhWRKY44 induced the expression of several defense-related genes in transgenic plants, including $P R-1, P R-2, P R-5$, and NPR1 in the SA pathway and $P R-4$ in the JA pathway, and enhanced the resistance of transgenic tobacco to $R$. solanacearum and Rhizoctonia solani $i^{51}$. After infected with Phytophthora nicotianae, the overexpression of SpWRKY1 enhanced the expression level of SA- and JA-associated genes (NtPR1, NtPR2, NtPR4, NtPR5, and NtPDF1.2), suggesting that SpWRKY1 acts as a positive regulator in tobacco defense responses to P. nicotianae ${ }^{52}$. The overexpression of NtPR-Q in tobacco enhanced the expression of SA dependent genes ( $N P R 1 a / c, N t P R 2$, and NtCHN50), thus enhanced the resistance of plants to R. solanacearum $^{53}$. In transgenic tobacco plants, the symptom of leaf wilting was lighter than that of wild type plants, and the expression levels of HR-associated gene HSR515 and SA-dependent marker genes NPR1 and PR2 were continuously upregulated by the ectopic overexpression of $\mathrm{CaC} 3 \mathrm{H} 14$, indicating that $\mathrm{CaC} 3 \mathrm{H} 14$ played a positive regulatory role in resistance to $R$. solanacearum ${ }^{54}$. In this study, the expression level of the NtPR2 gene was increased in the 35S::ScWRKY5 leaves at one-day post inoculation with R. solanacearum (Fig. 8B). Compared with the 35S::ScWRKY5, the leaves were yellow and the allergic spots were more serious in the control group after seven days of inoculation with $R$. solanacearum (Fig. 8A). These suggested that the transient overexpression of ScWRKY5 may enhance the resistance of $N$. benthamiana to $R$. solanacearum by participating in SA-dependent defense signaling pathways. After inoculation with F. solani var. coeruleum, the wilting disease symptoms were greater in 35S::ScWRKY5 leaves than in the control. Furthermore, the expression levels of the HR marker gene NtHSR515, and the SA pathway related genes NtPR-1a/c and NtPR2 were decreased in 35S::ScWRKY5 leaves (Fig. 8D). The results revealed that the transient overexpression of $S c W R K Y 5$ may reduce the resistance of $N$. benthamiana to F. solani var. coeruleum via repressed the expression of HR and SA pathway related genes. The regulation mechanism of the $S c W R K Y 5$ gene in disease resistance need to be further verified after stable genetic transformation. In summary, the ScWRKY5 gene may be involved in plant ABA, SA, and ET signal regulatory networks and plays a role in the plant response to biotic and abiotic stresses. However, more WRKY family genes in sugarcane need to be discovered and identified, which is more conducive to the further study of the possible functional differentiation between different family members of WRKY genes.

\section{Methods}

Plant materials and methods. In order to analyze the tissue-specific expression level of $S c W R K Y 5$, nine 10-month-old ROC22 (the main sugarcane variety in mainland China) plants were randomly selected from the field based on the method of Huang et al..$^{55}$. The sugarcane white root, bud, stem pith, stem epidermis, and +1 leaf were collected and frozen in liquid nitrogen, and then stored at $-80^{\circ} \mathrm{C}$ until use. Each sample contained three biological replicates, and each biological replicate contained three plants.

To detect the expression level of ScWRKY5 in sugarcane inoculated with smut pathogen, six different sugarcane varieties, including three smut-resistant varieties (YZ01-1413, LC05-136, and Yacheng05-179) and three smut-susceptible varieties (ROC22, YZ03-103, and FN40), were provided by the Key Laboratory of Sugarcane Biology and Genetics and Breeding, Ministry of Agriculture, Fujian Agriculture and Forestry University ${ }^{56}$. The 10 -month-old sugarcane stalks were selected and cultured at $32^{\circ} \mathrm{C}$. After the buds had germinated to $1-2 \mathrm{~cm}$ in length, an acupuncture inoculation method with $5 \times 10^{6} \mathrm{~mL}^{-1}$ (containing $0.01 \%$ Tween-20, $v / v$ ) smut pathogen suspension was used to inoculate the sugarcane buds, and the control group was inoculated with sterile water (containing $0.01 \%$ Tween-20, $v / v)^{57}$. All of the samples were cultured at $28^{\circ} \mathrm{C}$ for $16 \mathrm{~h}$ in the light and $8 \mathrm{~h}$ in the dark. Five single buds were collected at 0,1 , and $3 \mathrm{dpi}$, frozen in liquid nitrogen, and stored at $-80^{\circ} \mathrm{C}$ for future use.

For the analysis of the expression patterns of $S c W R K Y 5$ under different exogenous stresses, four-month-old ROC22 tissue culture seedlings were cultivated in water for about $10 \mathrm{~d}$. Two groups of the seedlings were cultured in aqueous solutions of $250 \mathrm{mM} \mathrm{NaCl}$ and $25 \%$ PEG 8000 solutions, and the leaves were sampled at $0,0.5,3$, 
6 , and $24 \mathrm{~h}$, respectively ${ }^{57-59}$. The leaves of the other three groups of the seedlings were sprayed with $5 \mathrm{mM}$ SA (with $0.01 \%$ Tween-20,v/v), $25 \mu \mathrm{M}$ MeJA (containing $0.1 \%$ ethanol and $0.05 \%$ Tween-20, $v / v$ ), and $100 \mu \mathrm{M} \mathrm{ABA \text {, }}$ respectively ${ }^{57-59}$. Under the SA, MeJA and ABA treatments, the leaves were collected at 0,3 , and $24 \mathrm{~h}$. All of the samples were frozen in liquid nitrogen and stored at $-80^{\circ} \mathrm{C}$ until use. Each sample contained three biological replicates, and each biological replicate contained three seedlings.

Total RNA extraction and cDNA first-strand synthesis. Total RNA of the sugarcane samples was extracted using the TRIzol method ${ }^{60}$. RQ1 RNase-Free DNase reagent was used to remove the DNA from the total RNA of the sample. The cDNA of the mature leaves of sugarcane ROC22, a template for ScWRKY5 gene cloning, was reverse-transcribed according to the RevertAid First Strand cDNA Synthesis Kit (Fermentas, Shanghai, China). Referring to the instruction manual of the Prime-Script RT Reagent Kit (TaKaRa Biotechnology, Dalian, China), the first-strand cDNA of the sugarcane tissues and different materials under various stresses was reversed and used to quantitatively detect the expression level of the target gene. The obtained RNA and cDNA were tested by $1.0 \%$ agarose gel electrophoresis.

Cloning, sequencing and bioinformatics analysis of ScWRKY5. From our previous transcriptome data of sugarcane ${ }^{61}$, a gene encoding a predictable WRKY transcription factor ScWRKY5 was screened. The National Center of Biotechnology Information (NCBI) online software (https://www.ncbi.nlm.nih.gov/tools/ primer-blast/) was used to design specific cloning amplification primers (Table S2). The cDNA of the mature leaves of ROC22 was used as a template, and a PCR reaction system was prepared according to the ExTaq DNA reagent instructions. The PCR program was set as $94^{\circ} \mathrm{C}$ pre-denaturation for $4 \mathrm{~min}$; followed by 35 cycles of $94^{\circ} \mathrm{C}$ denaturation for $30 \mathrm{~s}, 59.5^{\circ} \mathrm{C}$ (decreasing by $0.1^{\circ} \mathrm{C}$ in each cycle) for $30 \mathrm{~s}$, a $72^{\circ} \mathrm{C}$ extension for $2 \mathrm{~min}$; and then a final extension at $72{ }^{\circ} \mathrm{C}$ for another $10 \mathrm{~min}$. The PCR reaction product was detected by agarose gel electrophoresis, purified, and ligated to the cloning vector pMD19-T (TaKaRa Biotechnology, Dalian, China). The correct recombinant plasmid after sequencing (Fuzhou Shangya Biotechnology Co., Ltd.) was named pMD19T-ScWRKY5. ORF Finder (https://www.ncbi.nlm.nih.gov/orffinder/) and conserved domains database (CDD) (http://www.ncbi.nlm.nih.gov/Structure/cdd/wrpsb.cgi) ${ }^{62}$ were used to analyze the ORF of the ScWRKY5 gene and the conserved domain. The BLASTp program (https://blast.ncbi.nlm.nih.gov/Blast.cgi?PROGRAM=blast p\&PAGE_TYPE=BlastSearch\&LINK_LOC=blasthome) was used to find homologous amino acid sequences from other plant species. DNAMAN 6.0.3.99 software was used to perform sequence homology analysis. ProtParam (https://web.expasy.org/protparam/) ${ }^{63}$ and NPS@ server (https://npsa-prabi.ibcp.fr/cgi-bin/npsa_autom at.pl?page=/NPSA/npsa_hnn.html) ${ }^{64}$ were used to predict the primary structure and secondary structure of the target protein, respectively. Euk-mPLoc 2.0 Server (http://www.csbio.sjtu.edu.cn/bioinf/euk-multi-2/) ${ }^{65}$ and WoLF PSORT (https://wolfpsort.hgc.jp/) ${ }^{66}$ were both used for predicting the subcellular localization of the target protein. The neighbor-joining (NJ) (1000 bootstrap replicates) method in MEGA 7.0 software $^{67}$ was used to construct phylogenetic trees of ScWRKY5 protein and other WRKY proteins from S. bicolor ${ }^{68}$, Z. mays ${ }^{69}, S$. italica $^{18}, O$. sativa ${ }^{70}$, and S. spontaneum ${ }^{34}$. Nucleotide BLAST (https://blast.ncbi.nlm.nih.gov/Blast.cgi?PROGR AM=blastn\&PAGE_TYPE=BlastSearch\&LINK_LOC=blasthome) was used to extract the promoter sequence of the S. spontaneum WRKY gene (Sspon.03g0003780-2c). The Plant CARE (http://bioinformatics.psb.ugent.be/ webtools/plantcare $/ \mathrm{html} /)^{71}$ online software was used to predict the promoter element and function of the $S$. spontaneum WRKY gene (Sspon.03g0003780-2c).

Subcellular localization analysis. The Gateway entry vector primer pair ScWRKY5-Gate-F/R (Table S2) was designed based on the sequences of the ScWRKY5 ORF (without the stop codon) and the entry vector pDONR-221. After PCR amplification, the gel-purified product was subjected to the entry vector pDONR-221 by BP reaction to obtain a positive plasmid pDONR-221-ScWRKY5. Subsequently, after Ssp I single digestion and purification, $S c W R K Y 5$ was subjected to the subcellular localization vector $\mathrm{pMDC} 83^{72}$ by LR reaction. After sequencing, the recombinant subcellular localization vector $35 S:: S c W R K Y 5:: G F P$ was obtained.

The positive recombinant plasmid 35S::ScWRKY5::GFP was transformed into A. tumefaciens GV3101 strain. The Agrobacterium cells were collected and diluted to $\mathrm{OD}_{600}=0.8$ with MS blank medium. A 1.0-mL sterile syringe was used to transfer the bacterial solution (containing $200 \mu \mathrm{M}$ acetylsyringone) into the 5-8 leaves of $N$. benthamiana $a^{73,74}$. After culturing at $28^{\circ} \mathrm{C}$ for $16 \mathrm{~h}$ in the light and $8 \mathrm{~h}$ in the dark for $2 \mathrm{~d}$, the injected leaves were removed, and $1.0 \mu \mathrm{M} 4^{\prime}$, 6-diamidino-2-phenylindole (DAPI) was used for nuclear staining. A Leica TCS SP8 laser confocal microscope [Leica Microsystems (Shanghai) Trading Co., Ltd., Mannheim, Germany] with a $10 \times$ lens, $488 \mathrm{~nm}$ green fluorescence excitation wavelength, and $458 \mathrm{~nm}$ chromatin DAPI filter was used to observe the subcellular localization ${ }^{75,76}$.

Analysis of ScWRKY5 transcriptional self-activating activity. The target gene ScWRKY5 was constructed on the pGBKT7 vector, and the recombinant plasmid pGBKT7-ScWRKY5 was digested with enzymes and verified by sequencing analysis. A primer pair ScWRKY5-BD-F/R (Table S2) containing a specific restriction site was designed based on the sequences of ScWRKY5 ORF (without the stop codon) and the DNA-binding domain (BD) vector pGBKT7. The pMD19-T-ScWRKY5 plasmid was used as a template for PCR amplification. The gel-purified product and the pGBKT7 vector plasmid were digested with the Nde I and EcoR I enzymes, (Table S2) and then purified. Subsequently, the ScWRKY5 gene was ligated to the vector pGBKT7 by using T4 DNase, and transformed into DH5a competent cells. After bacterial liquid PCR detection, enzyme digestion verification, and sequencing comparison, the pGBKT7-ScWRKY5 recombinant plasmid was obtained.

The Y2HGold-GAL4 yeast two-hybrid system (containing four reporter genes AUR1-C, HIS3, ADE2, and $M E L 1)$ was used to analyze the transcriptional self-activating activity of ScWRKY5. According to the instructions 


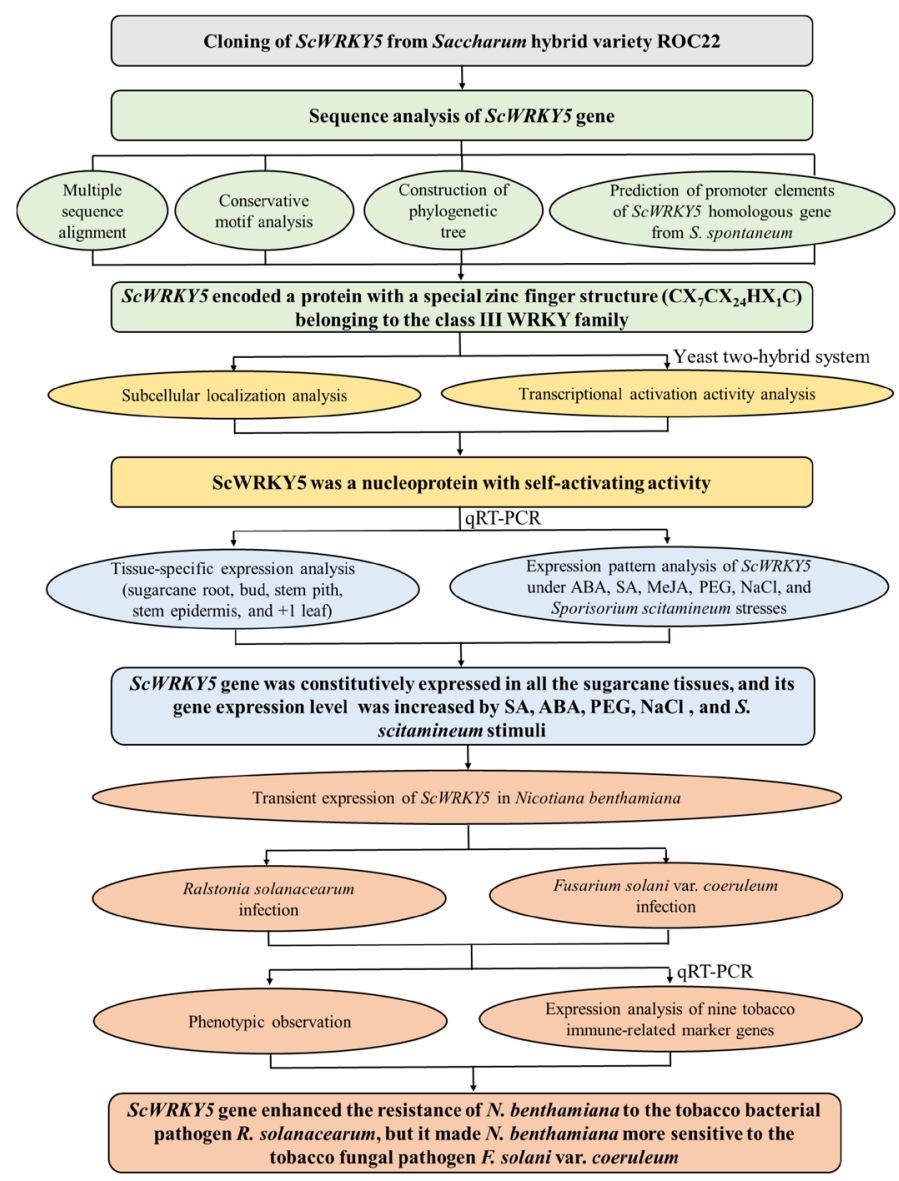

Figure 9. The mechanism-flow chart of the potential function of ScWRKY5.

of the Y2HGold Chemically Competent Cell, the positive hybridization vector plasmid pGBKT7-53 + pGADT7-T, the negative plasmid empty pGBKT7, and the constructed target fusion expression plasmid pGBKT7-ScWRKY5 were used to transform yeast Y2HGold competent cells in SDO (SD/-Trp, tryptophan-deficient medium) and cultured on a plate at $29^{\circ} \mathrm{C}$ for two days, until the bacteria grew. Then the bacteria solutions were diluted by $10^{-1}, 10^{-2}$, and $10^{-3}$, and $8.0 \mu \mathrm{L}$ each of them were spotted on SDO (SD/-Trp, tryptophan-deficient medium), $\mathrm{SDO} / \mathrm{X}$ (SD/-Trp/X- $\alpha$-Gal, tryptophan-deficient medium supplemented with 5-bromo-4-chloro-3-indole- $\alpha$-Dgalactoside), and SDO/X/A (SD/-Trp/X- $\alpha-G a l / A b A$, tryptophan-deficient medium supplemented with 5-bromo4-chloro-3-indole- $\alpha$-D-galactoside and astratin A) plates at $29^{\circ} \mathrm{C}$. The growing colonies were photographed, and the self-activation of the target protein was analyzed according to the growth status and color changes of the colonies.

Expression patterns of SCWRKY5 in sugarcane tissues and under various stresses. The 15-fold diluent cDNA of different sugarcane tissues (root, bud, leaf, stem pith, and stem epidermis) and samples treated with ABA, SA, MeJA, PEG, NaCl, and S. scitamineum was used as the qRT-PCR template. Based on the ScWRKY5 gene sequence, the specific qRT-PCR primer pair ScWRKY5-QF/R (Table S2) was designed on the NCBI online software. Glyceraldehyde-3-phosphate dehydrogenase (GAPDH, GenBank Accession No. CA254672) was used as the internal reference gene (Table S2). An ABI 7500 Real-Time PCR System (Applied Biosystems, Foster City, CA, USA) was used for qRT-PCR detection, and a quantitative reaction system was prepared according to the SYBR Green PCR Master Mix Kit instructions (Roche, Shanghai, China). Three technical replicates were set up for each sample, and sterile water was used as a template in the negative control. The relative expression of the target gene was calculated using $2^{-\Delta \Delta \mathrm{Ct}}$ method $^{76}$. The significance level of the data was analyzed using DPS 9.50 software, and graphs were plotted using Origin 8.0 software. In the smut pathogen infection test, the relative expression of the target gene was calculated with reference to the method of Su et al. ${ }^{77}$.

Transient expression of ScWRKY5 in N. benthamiana. After enzymatic digestion, the target fragment from the fusion entry vector pDONR221-ScWRKY5 was subjected to the overexpression vector pEarleyGate 203 by the Gateway LR Clonase II Enzyme mix (Invitrogen). After PCR verification, sequencing, and plasmid digestion, the correct recombinant overexpression vector pEarleyGate 203-ScWRKY5 was obtained. The empty vector pEarleyGate 203 and the recombinant vector pEarleyGate 203-ScWRKY5 were transformed into Agrobacterium 
GV3101. The Agrobacterium strain GV3101 carrying the recombinant plasmid was cultured in an Luria-Bertani (LB) liquid medium containing $50 \mu \mathrm{g} / \mathrm{mL}$ kanamycin and $35 \mu \mathrm{g} / \mathrm{mL}$ rifampicin at $200 \mathrm{rpm}$ and $28^{\circ} \mathrm{C}$. Agrobacterium cells cultured overnight were collected by centrifugation, resuspended in MS liquid medium containing $200 \mu \mathrm{M}$ acetylsyringone to $\mathrm{OD}_{600}=0.8$, and then the Agrobacterium cells were injected into 8-leaf-stage $N$. benthamiana leaves on the plate. The control was the Agrobacterium strain GV3101 cell carrying the pEarleyGate 203 empty vector ${ }^{73,74}$. After one day, the leaves were collected for qRT-PCR and DAB histochemical analysis. The tobacco bacterial pathogen $R$. solanacearum and fungal pathogen $F$. solani var. coeruleum were cultivated in potato dextrose water (PDW), diluted to $\mathrm{OD}_{600}=0.5$ with $10 \mathrm{mM}$ magnesium chloride $\left(\mathrm{MgCl}_{2}\right)$ solution, and inoculated into the overexpressing $N$. benthamiana leaves for one day. All of the treated plants were kept for a week under $28^{\circ} \mathrm{C}$ with a light period of $16 \mathrm{~h} / 8 \mathrm{~h}$ of darkness to track the changes in leaf symptoms and analyze the relative transcription levels of nine tobacco immune-related marker genes. The qRT-PCR was used to analyze the expression levels of the target gene and some tobacco immune marker-related genes in N. benthamiana, including the HR marker genes NtHSR201, NtHSR203 and NtHSR515, SA-related gene NtPR-1a/c, NtPR2 and $N t P R 3$, and ET synthesis-dependent genes NtEFE26 and NtAccdeaminase (Table S2) ${ }^{78,79}$. NtEF1- $\alpha$ (GenBank Accession No. D63396) was used as an internal reference (Table S2) $)^{78,79}$.

\section{Conclusions}

In the present study, a ScWRKY5 gene was isolated from sugarcane ROC22 and was found to encode a protein with a special zinc finger structure belonging to the class III WRKY family. ScWRKY5 was a nucleoprotein with self-activating activity. Through the prediction of the upstream promoter sequence of the S. spontaneum WRKY (Sspon.03g0003780-2c), as a homologue gene of ScWRKY5, several cis-acting elements involved in photosystem, hormone signaling substances, and abiotic stresses were found. The ScWRKY5 gene was constitutively expressed in different sugarcane tissues, and its expression level was increased under the stresses of SA, ABA, PEG, and $\mathrm{NaCl}$. After infected by S. scitamineum for $1 \mathrm{~d}$, the expression level of the $S c W R K Y 5$ gene was increased in two smut-resistant varieties (YZ01-1413 and LC05-136), while it was decreased in three smut-susceptible varieties (ROC22, YZ03-103, and FN40). Transient overexpression of the ScWRKY5 gene enhanced the defense ability of $N$. benthamiana to the tobacco bacterial pathogen $R$. solanacearum, but reduced the resistance response to the tobacco fungal pathogen F. solani var. coeruleum. Here, the potential function of ScWRKY5 in sugarcane was showed in a mechanism-flow chart (Fig. 9). These results may provide references for the excavation and functional identification of ScWRKY family genes in sugarcane.

Received: 12 June 2020; Accepted: 19 November 2020

Published online: 01 December 2020

\section{References}

1. Chinnusamy, V., Schumaker, K. \& Zhu, J. K. Molecular genetic perspectives on cross-talk and specificity in abiotic stress signalling in plants. J. Exp. Bot. 55, 225-236 (2004).

2. Rushton, P. J. \& Somssich, I. E. Transcriptional control of plant genes responsive to pathogens. Curr. Opin. Plant Biol. 1, 311-315 (1998).

3. Rushton, P. J., Somssich, I. E., Ringler, P. \& Shen, Q. J. WRKY transcription factors. Trends Plant Sci. 15, 247-258 (2010).

4. Martin, C. \& Paz-Ares, J. MYB transcription factors in plants. Trends Genet. 13, 67-73 (1997).

5. Olsen, A. N., Ernst, H. A., Leggio, L. L. \& Skriver, K. NAC transcription factors: Structurally distinct, functionally diverse. Trends Plant Sci. 10, 79-87 (2005).

6. Jakoby, M. et al. bZIP transcription factors in Arabidopsis. Trends Plant Sci. 7, 106-111 (2002).

7. Mizoi, J., Shinozaki, K. \& Yamaguchi-Shinozaki, K. AP2/ERF family transcription factors in plant abiotic stress responses. BBAGene Regul. Mec. 1819, 86-96 (2012).

8. Wang, K., Ding, Y., Cai, C., Chen, Z. \& Zhu, C. The role of $\mathrm{C} 2 \mathrm{H} 2$ zinc finger proteins in plant responses to abiotic stresses. Physiol. Plant. 165, 690-700 (2019).

9. Chen, X. et al. SQUAMOSA promoter-binding protein-like transcription factors: Star players for plant growth and development. J. Integr. Plant Biol. 52, 946-951 (2010).

10. Agarwal, P. K., Agarwal, P., Reddy, M. K. \& Sopory, S. K. Role of DREB transcription factors in abiotic and biotic stress tolerance in plants. Plant Cell Rep. 25, 1263-1274 (2006).

11. Renaumorata, B. et al. CDF transcription factors: Plant regulators to deal with extreme environmental conditions. J. Exp. Bot. 71, 3803-3815 (2020).

12. Eulgem, T., Rushton, P. J., Robatzek, S. \& Somssich, I. E. The WRKY superfamily of plant transcription factors. Trends Plant Sci. 5, 199-206 (2000).

13. Ishiguro, S. \& Nakamura, K. Characterization of a cDNA encoding a novel DNA-binding protein, SPF1, that recognizes SP8 sequences in the $5^{\prime}$ upstream regions of genes coding for sporamin and $\beta$-amylase from sweet potato. Mol. Gen. Genet. 244, 563-571 (1994).

14. Song, Y., Ai, C. R., Jing, S. J. \& Yu, D. Q. Research progress on functional analysis of rice WRKY genes. Rice Sci. 17, 60-72 (2010).

15. Pandey, S. P. \& Somssich, I. E. The role of WRKY transcription factors in plant immunity. Plant Physiol. 150, 1648-1655 (2009).

16. Mangelsen, E. et al. Phylogenetic and comparative gene expression analysis of barley (Hordeum vulgare) WRKY transcription factor family reveals putatively retained functions between monocots and dicots. BMC Genom. 9, 194 (2008).

17. Wei, K. F., Chen, J., Chen, Y. F., Wu, L. J. \& Xie, D. X. Molecular phylogenetic and expression analysis of the complete WRKY transcription factor family in maize. DNA Res. 19, 153-164 (2012).

18. Muthamilarasan, M. et al. Global analysis of WRKY transcription factor superfamily in Setaria identifies potential candidates involved in abiotic stress signaling. Front. Plant Sci. 6, 910 (2015).

19. Dong, J., Chen, C. \& Chen, Z. Expression profiles of the Arabidopsis WRKY gene superfamily during plant defense response. Plant Mol. Biol. 51, 21-37 (2003).

20. Bakshi, M. \& Oelmüller, R. WRKY transcription factors: Jack of many trades in plants. Plant Signaling Behav. 9, e27700 (2014).

21. Qiu, Y. P., Jing, S. J., Fu, J., Li, L. \& Yu, D. Q. Cloning and analysis of expression profile of 13 WRKY genes in rice. Chin. Sci. Bull. 49, 2159-2168 (2004). 
22. Rushton, P. J. et al. Interaction of elicitor-induced DNA-binding proteins with elicitor response elements in the promoters of parsley PR1 genes. EMBO. J. 15, 5690-5700 (1996).

23. Liu, D. L., Leib, K., Zhao, P. Y., Kogel, K. H. \& Langen, G. Phylogenetic analysis of barley WRKY proteins and characterization of HvWRKY1 and-2 as repressors of the pathogen-inducible gene HvGER4c. Mol. Genet. Genom. 289, 1331-1345 (2014).

24. Ramamoorthy, R., Jiang, S. Y., Kumar, N., Venkatesh, P. N. \& Ramachandran, S. A comprehensive transcriptional profiling of the WRKY gene family in rice under various abiotic and phytohormone treatments. Plant Cell Physiol. 49, 865-879 (2008).

25. Wu, J., Chen, J. B., Wang, L. F. \& Wang, S. M. Genome-wide investigation of WRKY transcription factors involved in terminal drought stress response in common bean. Front. Plant Sci. 8, 380 (2017).

26. Jiang, Y. Z. et al. Genome-wide identification and characterization of the Populus WRKY transcription factor family and analysis of their expression in response to biotic and abiotic stresses. J. Exp. Bot. 65, 6629-6644 (2014).

27. Zhang, T., Tan, D. F., Zhang, L., Zhang, X. Y. \& Han, Z. X. Phylogenetic analysis and drought-responsive expression profiles of the WRKY transcription factor family in maize. Agri Gene. 3, 99-108 (2017).

28. de Souza, A. P., Grandis, A., Leite, D. C. C. \& Buckeridge, M. S. Sugarcane as a bioenergy source: History, performance, and perspectives for second-generation bioethanol. Bioenergy Res. 7, 24-35 (2014).

29. Li, Y. R. \& Yang, L. T. Sugarcane agriculture and sugar industry in China. Sugar Tech. 17, 1-8 (2015).

30. Liu, J. X. et al. Molecular cloning and expression analysis of a WRKY transcription factor in sugarcane. Afr. J. Biotechnol. 11, 6434-6444 (2012).

31. Wang, L. et al. Expression characteristics and functional analysis of the ScWRKY3 gene from sugarcane. Int. J. Mol. Sci. 19, 4059 (2018).

32. Wang, L. et al. Cloning and expression characteristic analysis of ScWRKY4 gene in sugarcane. Acta Agron. Sin. 44, 1367-1379 (2018).

33. Zhang, X. et al. Cloning and expression analysis of a IId sub-group WRKY transcription factor gene from sugarcane. Sci. Agric. Sin. 51, 4409-4423 (2018).

34. Li, Z. et al. Genome-wide identification and expression profile analysis of WRKY family genes in the autopolyploid Saccharum spontaneum. Plant Cell Physiol. 6, 616-630 (2019).

35. Huang, S. et al. Genome-wide analysis of WRKY transcription factors in Solanum lycopersicum. Mol. Genet. Genomics. 287, 495-513 (2012).

36. Silhavy, T. J., Benson, S. A. \& Emr, S. D. Mechanisms of protein localization. Microbiol. Rev. 47, 313-344 (1983).

37. Xiang, X. H. et al. Genome-wide identification and expression analysis of the WRKY gene family in common tobacco (Nicotiana tabacum L.). Hereditas. 38, 840-856 (2016).

38. Wang, H. H. et al. Rice WRKY4 acts as a transcriptional activator mediating defense responses toward Rhizoctonia solani, the causing agent of rice sheath blight. Plant Mol. Biol. 89, 157-171 (2015).

39. Fields, S. \& Song, O. K. A novel genetic system to detect protein-protein interactions. Nature 340, 245-246 (1989).

40. Besseau, S., Li, J. \& Palva, E. T. WRKY54 and WRKY70 co-operate as negative regulators of leaf senescence in Arabidopsis thaliana. J. Exp. Bot. 63, 2667-2679 (2012).

41. Wang, X. et al. GhWRKY40, a multiple stress-responsive cotton WRKY gene, plays an important role in the wounding response and enhances susceptibility to Ralstonia solanacearum infection in transgenic Nicotiana benthamiana. PLoS ONE 9, e93577 (2014).

42. Wan, Y. Q. et al. Identification of the WRKY gene family and functional analysis of two genes in Caragana intermedia. BMC Plant Biol. 18, 31 (2018).

43. Wang, X. T. et al. Expression of TaWRKY44, a wheat WRKY gene, in transgenic tobacco confers multiple abiotic stress tolerances. Front. Plant Sci. 6, 615 (2015).

44. Zhou, L. et al. Molecular characterization of 26 cotton WRKY genes that are expressed differentially in tissues and are induced in seedlings under high salinity and osmotic stress. Plant Cell Tiss Organ Cult. 119, 141-156 (2014).

45. Hara, K., Yagi, M., Kusano, T. \& Sano, H. Rapid systemic accumulation of transcripts encoding a tobacco WRKY transcription factor upon wounding. Mol. Gen. Genet. 263, 30-37 (2000).

46. Bartels, D. \& Sunkar, R. Drought and salt tolerance in plants. Crit. Rev. Plant Sci. 24, 23-58 (2005).

47. Gao, Y. F. et al. The WRKY transcription factor WRKY8 promotes resistance to pathogen infection and mediates drought and salt stress tolerance in Solanum lycopersicum. Physiol. Plant. 168, 98-117 (2020).

48. Chen, X. T. et al. Overexpression of AtWRKY28 and AtWRKY75 in Arabidopsis enhances resistance to oxalic acid and Sclerotinia sclerotiorum. Plant Cell Rep. 32, 1589-1599 (2013).

49. Qiu, D. Y. et al. Rice gene network inferred from expression profiling of plants overexpressing OsWRKY13, a positive regulator of disease resistance. Mol. Plant. 1, 538-551 (2008).

50. Mukhtar, M. S., Deslandes, L., Auriac, M., Marco, Y. \& Somssich, I. E. The Arabidopsis transcription factor WRKY27 influences wilt disease symptom development caused by Ralstonia solanacearum. Plant J. 56, 935-947 (2008).

51. Li, J., Wang, J., Wang, N. X., Guo, X. Q. \& Gao, Z. GhWRKY44, a WRKY transcription factor of cotton, mediates defense responses to pathogen infection in transgenic Nicotiana benthamiana. Plant Cell Tiss. Organ Cult. 121, 127-140 (2015).

52. Li, J. B., Luan, Y. S. \& Liu, Z. Overexpression of SpWRKY1 promotes resistance to Phytophthora nicotianae and tolerance to salt and drought stress in transgenic tobacco. Physiol. Plant. 155, 248-266 (2015).

53. Tang, Y. M., Liu, Q. P., Liu, Y., Zhang, L. L. \& Ding, W. Overexpression of NtPR-Q up-regulates multiple defense-related genes in Nicotiana tabacum and enhances plant resistance to Ralstonia solanacearum. Front. Plant Sci. 8, 1963 (2017).

54. Qiu, A. et al. $\mathrm{CaC} 3 \mathrm{H} 14$ encoding a tandem $\mathrm{CCCH}$ zinc finger protein is directly targeted by CaWRKY 40 and positively regulates the response of pepper to inoculation by Ralstonia solanacearum. Mol. Plant Pathol. 19, 2221-2235 (2018).

55. Huang, N. et al. Cloning and expression analysis of a diaminopimelate epimerase gene in sugarcane. Chin. J. Trop. Crops. 34, 2200-2208 (2013).

56. Su, Y. C. et al. Early selection for smut resistance in sugarcane using pathogen proliferation and changes in physiological and biochemical indices. Front. Plant Sci. 7, 1133 (2016).

57. Su, Y. C. et al. Isolation of a novel peroxisomal catalase gene from sugarcane, which is responsive to biotic and abiotic stresses. PLOS ONE. 9, e884426 (2014).

58. Li, H. et al. ZmWRKY33, a WRKY maize transcription factor conferring enhanced salt stress tolerances in Arabidopsis. Plant Growth Regul. 70, 207-216 (2013).

59. Scarpeci, T. E., Zanor, M. I., Mueller-Roeber, B. \& Valle, E. M. Overexpression of AtWRKY30 enhances abiotic stress tolerance during early growth stages in Arabidopsis thaliana. Plant Mol. Biol. 83, 265-277 (2013).

60. Connolly, M. A., Clausen, P. A. \& Lazar, J. G. Preparation of RNA from plant tissue using trizol. Cold Spring Harb. Protoc. 2006, 4105 (2006).

61. Yang, Y. T. et al. Identification of cold-related miRNAs in sugarcane by small RNA sequencing and functional analysis of a cold inducible ScmiR393 to cold stress. Environ. Exp. Bot. 155, 464-476 (2018).

62. Marchler-Bauer, A. et al. CDD/SPARCLE: Functional classification of proteins via subfamily domain architectures. Nucleic Acids Res. 45, D200-D203 (2017).

63. Gasteiger, E. et al. Protein identification and analysis tools on the ExPASy server. In The Proteomics Protocols Handbook 571-607 (Springer, Berlin, 2005). 
64. Combet, C., Blanchet, C., Geourjon, C. \& Deleage, G. NPS@: Network protein sequence analysis. Trends Biochem. Sci. 25, 147-150 (2000).

65. Chou, K. C. \& Shen, H. B. A new method for predicting the subcellular localization of eukaryotic proteins with both single and multiple sites: Euk-mPLoc 2.0. PLoS ONE. 5, e9931 (2010).

66. Horton, P. et al. WoLF PSORT: Protein localization predictor. Nucleic Acids Res. 35, W585-W587 (2007).

67. Kumar, S., Stecher, G. \& Tamura, K. MEGA7: molecular evolutionary genetics analysis version 7.0 for bigger datasets. Mol. Biol. Evol. 33, 1870-1874 (2016).

68. Ahmadi, J., Agahi, K. \& Fabriki Ourang, S. Bioinformatics genome-wide characterization of the WRKY gene family in Sorghum bicolor. Iran. J. Genet. Pl. Br. 8, 33-45 (2019).

69. Wei, K. F., Chen, J., Chen, Y. F., Wu, L. J. \& Xie, D. X. Multiple-strategy analyses of ZmWRKY subgroups and functional exploration of ZmWRKY genes in pathogen responses. Mol. Biosyst. 8, 1940-1949 (2012).

70. Xie, Z. et al. Annotations and functional analyses of the rice WRKY gene superfamily reveal positive and negative regulators of abscisic acid signaling in aleurone cells. Plant Physiol. 137, 176-189 (2005).

71. Lescot, M. et al. PlantCARE, a database of plant cis-acting regulatory elements and a portal to tools for in silico analysis of promoter sequences. Nucleic Acids Res. 30, 325-327 (2002).

72. Curtis, M. D. \& Grossniklaus, U. A gateway cloning vector set for high-throughput functional analysis of genes in planta. Plant Physiol. 133, 462-469 (2003).

73. Choi, D. S., Hwang, I. S. \& Hwang, B. K. Requirement of the cytosolic interaction between pathogenesis-related PROTEIN10 and leucine-rich repeat PROTEIN1 for cell death and defense signaling in pepper. Plant Cell. 24, 1675-1690 (2012).

74. Fan, Z. Q. et al. The banana transcriptional repressor MaDEAR1 negatively regulates cell wall-modifying genes involved in fruit ripening. Front. Plant Sci. 7, 1021 (2016).

75. Liu, X. F. et al. GhWRKY25, a group I WRKY gene from cotton, confers differential tolerance to abiotic and biotic stresses in transgenic Nicotiana benthamiana. Protoplasma. 253, 1265-1281 (2016).

76. Livak, K. J. \& Schmittgen, T. D. Analysis of relative gene expression data using real-time quantitative PCR and the $2^{-\Delta \Delta C T}$ method. Methods. 25, 402-408 (2001).

77. Su, Y. C. et al. Molecular cloning and functional identification of peroxidase gene ScPOD02 in sugarcane. Acta Agron. Sin. 43, 510-521 (2017).

78. Lai, Y. et al. Overexpression of a Chinese cabbage BrERF11 transcription factor enhances disease resistance to Ralstonia solanacearum in tobacco. Plant Physiol. Biochem. 62, 70-78 (2013).

79. Peng, Q. et al. A sugarcane pathogenesis-related protein, ScPR10, plays a positive role in defense responses under Sporisorium scitamineum, SrMV, SA, and MeJA stresses. Plant Cell Rep. 36, 1427-1440 (2017).

\section{Acknowledgements}

This research was funded by the National key R\&D Program of China (2018YFD1000503 and 2019YFD1000500), the National Natural Science Foundation of China (31871688, 31671752, 31101196 and 31340060), the Natural Science Foundation of Fujian Province (2020J01591) and the China Agriculture Research System (CARS-17).

\section{Author contributions}

Y.Q. and Y.S. conceived and designed the research. D.W. and L.W. prepared the materials. D.W., L.W., W.S., Y.R., C.Y., and C.Z. conducted the experiments. D.W., L.W., W.S., and Y.R. analyzed the data. D.W. wrote the manuscript. Y.Q. and Y.S. helped to revise the manuscript. All authors read and approved the final manuscript.

\section{Competing interests}

The authors declare no competing interests.

\section{Additional information}

Supplementary information is available for this paper at https://doi.org/10.1038/s41598-020-78007-9.

Correspondence and requests for materials should be addressed to Y.Q. or Y.S.

Reprints and permissions information is available at www.nature.com/reprints.

Publisher's note Springer Nature remains neutral with regard to jurisdictional claims in published maps and institutional affiliations.

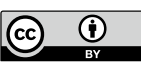

Open Access This article is licensed under a Creative Commons Attribution 4.0 International License, which permits use, sharing, adaptation, distribution and reproduction in any medium or format, as long as you give appropriate credit to the original author(s) and the source, provide a link to the Creative Commons licence, and indicate if changes were made. The images or other third party material in this article are included in the article's Creative Commons licence, unless indicated otherwise in a credit line to the material. If material is not included in the article's Creative Commons licence and your intended use is not permitted by statutory regulation or exceeds the permitted use, you will need to obtain permission directly from the copyright holder. To view a copy of this licence, visit http://creativecommons.org/licenses/by/4.0/.

(C) The Author(s) 2020 